\title{
Nano-functionalized paper-based loT enabled devices for point-of-care testing: a review
}

\author{
Vinay Kishnani ${ }^{1} \cdot$ Sungjune Park ${ }^{2} \cdot$ Umesh T. Nakate ${ }^{2} \cdot$ Kunal Mondal $^{3} \cdot$ Ankur Gupta $^{1}$ (D)
}

Accepted: 20 September 2021 / Published online: 18 November 2021

(c) The Author(s), under exclusive licence to Springer Science+Business Media, LLC, part of Springer Nature 2021

\begin{abstract}
Over the last few years, the microfluidics phenomenon coupled with the Internet of Things (IoT) using innovative nanofunctional materials has been recognized as a sustainable and economical tool for point-of-care testing (POCT) of various pathogens influencing human health. The sensors based on these phenomena aim to be designed for cost-effectiveness, make it handy, environment-friendly, and get an accurate, easy, and rapid response. Considering the burgeoning importance of analytical devices in the healthcare domain, this review paper is based on the gist of sensing aspects of the microfabricated paper-based analytical devices ( $\mu$ PADs). The article discusses the various used design methodologies and fabrication approaches and elucidates the recently reported surface modification strategies, detection mechanisms viz., colorimetric, electrochemical, fluorescence, electrochemiluminescence, etc. In a nutshell, this article summarizes the state-of-the-art research work carried out over the nano functionalized paper-based analytical devices and associated challenges/solutions in the point of care testing domain.
\end{abstract}

Keywords Sensor $\cdot$ IoT, $\mu$ PADs $\cdot$ Sensitivity $\cdot$ Point of care testing $\cdot$ Selectivity

\section{Introduction}

Initial screening of diseases at the preliminary level is still a challenge across the globe, and in this context, microfabricated analytical devices on paper substrates have been visualized as one of the sustainable and commercially viable solutions in the point of care testing (POCT) domain due to their portability, economy, easy availability, and transportability. Paper-based sensors have recently experienced great progression in terms of their properties and are fulfilling the ASSURED law: viz.(1) Affordable (2) Sensitive (3) Specific (4) Users friendly (5) Rapid and Robust (6) Equipment Free (7) Deliverable to all end users (Nilghaz et al. 2015, 2016). In the aforementioned specifications, sensitivity plays a crucial role in the quality of the devices. Analytical devices aimed at POCT can be improved

Ankur Gupta

ankurgupta@iitj.ac.in

1 Department of Mechanical Engineering, Indian Institute of Technology Jodhpur-342037, Rajasthan, India

2 Department of Polymer Nano Science and Technology, Jeonbuk National University, Jeonju 54896, South Korea

3 Materials Science and Engineering Department, Idaho National Laboratory, Idaho Falls ID 83415, USA via basic modifications in conventional detection techniques for high-performance sensitivity. The available conservative detection techniques are electrochemical detection, colorimetric detection, chemiluminescence, fluorescence, mass spectrum, and surface-enhanced Raman spectroscopy, etc. (Gopinath et al. 2016). The device sensitivity can also be tuned by materials engineering, surface modification, change in design, and fabrication technique. As an example, the detection process can be enhanced by incorporating active catalysts such as silver nanoparticles (Ag NPs), gold nanoparticles (Au NPs), metal oxide nanoparticles, bi, and tri-metallic nanoparticles, hyperbranched polymers, graphene and its derivatives, nanoparticles incorporated carbon-based materials, quantum dots, up-conversion nanoparticles, etc. Enhancement in the sensor's surface and detection affects numerous technical and viable specifications of the device. These specifications are LOD, specificity, assay speed, detection format, sample matrices and packaging, labeling, stability requirements, and the target cost. The detection sensitivity is often calculated by dividing the slope of the linear range curve by the surface area using the projection area. The different methods designed for different analytes vary in size, function, and LOD. The functionality of $\mu$ PADs is broad and can be used for various purposes, for initial on-site screening of human health, water, food, and air. With minute 
modification in the surface of $\mu$ PADs, various diseases have been reported to be diagnosed and heavy metal ions in air and water, toxic gases/chemicals in air/water, food/beverages quality, etc. The quantification of analytes improved through the integration of electrochemical sensors or strip readers, and low detection limits and higher sensitivity were achieved through these readers and sensors. Further novel and multi detection of analytes, improved analytical performance, stability, and higher shelf life can be achieved by integrating nanomaterials in paper-based surface (Parolo and Merkoçi 2013). The sustainability of the lateral flow biosensors (LFB), which is a kind of paper-based biosensor, can be enhanced through the integration of new nano materials, methods, and strategies. The higher sensitivity and lower detection limit can be achieved through the (1) nanoparticles, which are stable, easy to detect, and provides intense signals (2) developing strategies, which increases signaling quantity per unit of analytes (3) combination of different detection methods, which removes drawbacks of the individual detection system and provides high quantification and strong signal in detection zone (Quesada-González and Merkoçi 2015).There is a lot of advancement in the microfluidic system for emergency analysis of environmental (soil, air, ecology, river water) (Kung et al. 2019), foodborne pathogens (Al Mughairy and Al-Lawati 2020; Puiu and Bala 2020). Apart from modifications in design, and surface property enhancement of $\mu \mathrm{PADs}$, reporting system also plays a crucial role in the sensing mechanism of the POCT system. Reporting system basically depends on the sensing mechanism of the $\mu$ PADs. It can be numeric, image, digital data, graph, etc. After the assays, it is important to read out the information generated through the optical or electrochemical measurement. It is a significant step for equipment dependent quantitative readouts. But, as per the requirements semi-quantitative and qualitative read outs can be performed. The information gathered can be utilized through a standard analytical-chemical instrumental technique viz., diffuse reflectance spectroscopy, IT communication equipment or electronic equipments such as cell phones, scanners etc.(Quesada-González and Merkoçi 2017). Smartphones now a days are ubiquitous and are reported to play an important role in performing POCT in sustainable and economical fashion. They not only persist analytical reading capabilities through the screen which can act as a display as well as controller, but also a potential source of capturing the signals via inbuilt camera, audio jack, power port, and ambient light sensors. They are well known medium of a huge storage (device and cloud storage) for data collection and its analysis capability through various software, as well as data transfer ability through wire or wireless (Bluetooth, NFC, Wi-Fi etc.) system (Jena et al. 2019). These devices act as a part of internet of things (IoT). Through internet connections, colorimetric images/ electronic signals can be compared with data samples available in data base and an accurate data interpretation can be made available in no time. Machine learning (ML) and artificial intelligence
(AI) have been proven to be better tools to predict the patterns via comparing previous data available in the data base. This is very much helpful for the analysis of human health, water, food/ beverages, and environmental conditions. Regarding the signal measurement, data transfer, and data analysis, fifth generation $(5 \mathrm{G})$ smart-phones have very high capabilities. It can detect visual stimulation at high sensitivity, and image resolution through a high definition (HD) camera, as well as fast data interpretation through high internet and fast processor.

Paper-based sensing devices fulfilled all the criteria of ASSURED law and provides sustainable solutions. It is economical, environment friendly, and easy to operate at end users. It is easily applicable for all the diagnostic assays by integrating electrical and optical sensing modalities. It is demonstrated that it can detect and isolate various bio-targets and pathogens in different matrices, including plasma, whole blood, and peritoneal dialysis fluid (Shafiee et al. 2015). An android app is developed for reading the information generated during testing of blood type in a paper-based sensor. The barcode-like design was developed for easier analysis of length-based information through the smartphone, and it is reported that there is less environment effects in comparison to the electrical and colorimetric signals. The results generated were saved as electronic data and transfer through the text message to the patient/professionals (Guan et al. 2014).

Further, Moazeni et al. (Moazeni et al. 2018) developed a novel peptide modified plastic-paper $\mu$ PADs for the detection of alpha-fetoprotein (AFP) in human serum. The upper surface of chromatography paper modified with the silvergraphene electrode (20\% Ag) and diphenylalanine was used in the detection zone to increase the sensitivity and stability of the immobilized antibody. It showed detection limit 1 and $10 \mathrm{ngmL}^{-1}$ in Phosphate Buffer Saline (PBS) and plasma, respectively. Adkins et al. (Adkins et al. 2017) developed a method for recognizing fecal indicator bacteria (FIB) through electrochemical and colorimetric detection, and two different methods utilize to overcome the disadvantage of a particular method. It has resulted that the detection limit of the colorimetric method is higher in comparison to the electrochemical method while the detection time was the same. Overall, the development of point-of-care testing technology needs not only the automation in manual activities but also, structured data collection, monitoring and planning to support the existing clinical decision system, as represented in the Fig. 1.

\section{Design layouts and surface modifications in paper-based analytical devices}

As per the research in the last few years, it is observed the nanomaterials play a vital role in enhancing the electrode surface and bio sensing paper device performance by enhancing analytical signal with the improved available superficial area 


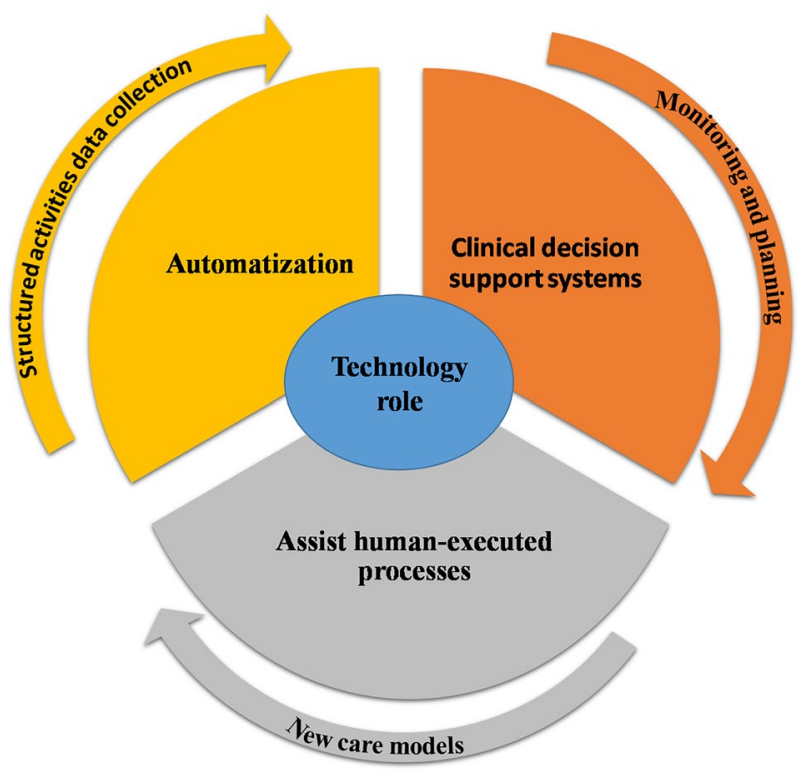

Fig. 1 Role of technology in point of care testing

of $\mu$ PADs. The modification of the paper surface addresses the problems associated with the lack of homogeneity on color measurements that compromise the sensitivity and detectability in clinical diagnosis. Figure 2 demonstrates the modules explored in paper-based devices for detection.

\subsection{Types of paper}

Among various forms of paper available commercially, selection for the paper for sensors and diagnostic devices depends on its surface engineering aspects and corresponding application. While choosing a paper for fabricating a paper-based device, various properties must be analyzed as per the final application, viz. wicking ability, flow rate, thickness, pore size, and retention rate. For $\mu$ PADs, blotting, filter, and chromatography papers are used widely, further Whatman brand chromatography paper is used at a vast scale due to its higher wicking ability and low pore size at the same time. Whatman-4 is used for distinct applications due

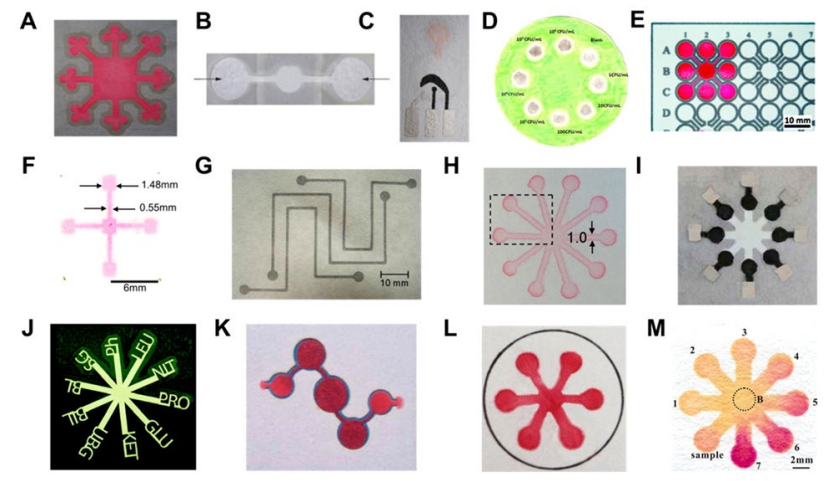

Fig. 2 Design modulus explored in paper-based devices for detection Reproduced with kind permission from Cate et al. (2015) to its large pore size, and higher retention rate. Other types of chemical and/or physical properties are required as per the target application. As an example, nitrocellulose membranes are frequently used due to their chemical functional group, which allows covalent immobilization of the biomolecules, weak hydrogen bonds, charge to charge interaction, and interaction of protein-based substrates through van der Waals interaction. Due to its higher protein binding ability, nitrocellulose membranes are frequently used for the Au NP-based assay and ELISA. Table 1 represents the various type of papers with its physical properties (Healthcare Systems Home I GE Healthcare I GE Healthcare (India) 2021; Advantec MFS 2021; Ahlstrom-Munksjö - Other specialty papers 2021).

\subsection{Design layouts}

Fluid in the paper-based substrate is transported through the capillary action which is due to the relationship between adhesive and cohesive force. Transportation of fluid in the paper can be explained through the Lucas-Washburn equation. It describes the one-dimensional capillary flow in a parallel cylindrical tubes bundle (Washburn 1921). The relation between wicking time and wetted length can be explained through Eq. 1 for ideal condition, whereas simultaneous flow rate in the paper can be expressed as Eq. 2 (Fu et al. 2011), where, 1(t) is the distance penetrated by a liquid flowing under capillary pressure into a channel, $\gamma$ is the surface tension of the liquid, $r$ is average pore radius also called as effective pore radius, $\theta$ is the contact angle between the fluid and boundary wall, $\mu$ is viscosity and $t$ is time, $Q$ represents the volumetric flow rate, $\mathrm{k}$ is the permeability of the paper to the fluid, $\mathrm{A}$ is the cross-sectional area of the channel perpendicular to the flow, $\mathrm{A}=\mathrm{W}^{*} \mathrm{H}(\mathrm{W}=$ width,

Table 1 Frequently used paper substrates for $\mu$ PADs with their properties

\begin{tabular}{|c|c|c|c|c|}
\hline Name & $\begin{array}{l}\text { Pore } \\
\text { diameter } \\
(\mu \mathrm{m})\end{array}$ & $\begin{array}{l}\text { Initial filtration } \\
\text { speed } \\
\text { (Herzberg) }\end{array}$ & $\begin{array}{l}\text { Thickness } \\
(\mathrm{mm})\end{array}$ & $\begin{array}{l}\text { Weight } \\
\left(\mathrm{g} / \mathrm{m}^{2}\right)\end{array}$ \\
\hline Whatman 1 & 11 & 150 & 0.18 & 87 \\
\hline Whatman 3 & 6 & 325 & 0.39 & 185 \\
\hline $\begin{array}{l}\text { Whatman } 3 \\
\text { MM }\end{array}$ & 6 & N/A & 0.34 & 189 \\
\hline Whatman 4 & $20-25$ & 37 & 0.20 & 92 \\
\hline Whatman 113 & 30 & 28 & 0.42 & 125 \\
\hline Whatman 114 & 25 & 38 & 0.19 & 77 \\
\hline Whatman 41 & $20-25$ & 54 & 0.22 & 85 \\
\hline Whatman 54 & 22 & 39 & 0.18 & 90 \\
\hline Advantec 1 & N/A & 45 & 0.20 & 90 \\
\hline Advantec 2 & N/A & 80 & 0.26 & 125 \\
\hline Ahlstrom 319 & N/A & 72 & 0.48 & 180 \\
\hline
\end{tabular}


$\mathrm{H}=$ Height), $\Delta \mathrm{P}$ is the pressure difference along the direction of flow over the length $\mathrm{L}$ being characteristic length scale. The volumetric fluid flow rate varies with respect to the available number of sections and geometry available in a paper-based scheme and can be expressed as Eq. 3. But for application purposes, paper-based schemes are used in ambient circumstances where evaporation affects the transportation of fluid, so the (Eq. 1) can be better transformed to the Eq. 4 (Camplisson et al. 2015). The modified form of Eq. 1 is used for the fluid transportation in paper-based scheme for non-uniform cross-section by predicting filling time $t$ for an arbitrary cross-section $\{\mathrm{A}(\mathrm{l})\}$ and wetted distance $\{1(\mathrm{t})\}$ (Elizalde et al. 2015), can be expressed through Eq. 5. The reported effect on contact angle variation due to the hydrophobic barriers is explained through modification in Eq. 1 and can be expressed through the Eq. 6 (Hong and Kim 2015).

$$
\begin{aligned}
& \mathbf{l}(\mathbf{t})=\sqrt{\frac{\gamma \mathbf{r c o s} \theta}{2 \mu} \mathbf{t}} \\
& \mathbf{Q}=-\frac{\mathbf{k W H}}{\mu \mathbf{L}} \Delta \mathbf{P} \\
& \mathbf{Q}=-\frac{\Delta P}{\sum_{i}^{n} \frac{\mu L_{i}}{k W_{i} H_{i}}} \\
& \mathbf{l}(\mathbf{t})=\sqrt{\frac{\gamma \mathbf{r} \phi \mathbf{h} \cos \theta}{4 \mu \mathbf{q}_{0}}\left(1-\mathbf{e}^{-\frac{2 \mathbf{q}_{0}}{\phi \mathbf{h}} \mathbf{t}}\right)} \\
& \mathrm{Dt}=\frac{\boldsymbol{k} \boldsymbol{R}_{0}}{\mu} \int_{0}^{l} A\left(l^{\prime}\right) d l^{\prime}+\int_{0}^{l}\left[A\left(l^{\prime}\right) \int_{0}^{l^{\prime}} \frac{d x}{A(x)}\right] d l^{\prime} \\
& \mathbf{l}(\mathbf{t})=\mathbf{k} \sqrt{\left(1+\boldsymbol{\beta} \frac{\mathbf{d}}{\phi^{\frac{1}{3}} \mathbf{w}} \frac{\cos \theta_{\mathbf{b}}}{\cos \theta}\right) \frac{\sigma}{\mu} \mathbf{t}}
\end{aligned}
$$

Where, $L_{i}$ is the length of the segment $i$ of the channel in the direction of flow, $\mathrm{n}$ is the number of connected straight channels, $\mathrm{k}$ represents the permeability of the paper to the fluid, $\varnothing$ is the porosity of the paper, $h$ is the thickness of the porous channel, $\mathrm{q}_{0}$ is the rate of evaporation and $\mathrm{D}(=\mathrm{k} \Delta \mathrm{p} / \mu)$ is the diffusive coefficient.

(Nguyen et al. 2018) proposed that the performance and sensitivity of the $\mu$ PADs could be increased by a simple change in the representative structure of $\mu$ PADs, such as enlarging the detection zone, adding a waste zone, and using an elution step, that can reduce sample loss. The optimized $\mu$ PADs showed a $28 \%$ increase in sensitivity and a $78 \%$ increase in maximum signal for $\mathrm{Ni}$ (II) samples, and a $94 \%$ increase in the maximum signal for Mn (11). (Yang et al. 2019) designed five different structures of the paper microfluidic chip to find image grey level statistics for chromogenic reaction and designed Y-Channel, three-channel, rotary array, oblique double channel, and multilayer solid microfluidic chip and concluded that no significant relationship between the color reaction uniformity and the number of channels but found a positive correlation with the reaction contact area. In other work, (Li et al. 2016) used origami paper-based analytical devices to detect glucose through the three-electrode system and the electrode prepared using direct drawing of a pencil. It (origami paper) showed excellent analytical performance for glucose sensing, reproducibility, and selectivity for common interferents in physiological fluids and exhibited LOD and linear range of $0.05 \mathrm{mM}$ and 1-12 mM, respectively. (Park and Park 2017) modified a 3D paper-based microfluidic device by utilizing a pressed paper for the detection of c-reactive protein in vertical flow multistep assay, which produced delayed flow and programmed delivery order of the reagents and detected highly sensitive c-reactive protein within $15 \mathrm{~min}$ with increased sensitivity and the upper limit of the assays, shows that vertical flow assays with multistep reaction would be much beneficial than lateral flow assays for comprehensive range detection of target analytes. (Zhou 2020) used 3D rotary $\mu$ PADs containing three layers of paper-based chip and its component, the top layer rotates at $90^{\circ}$ to facilitate the detection window on the top layer corresponded to the paper@ZnSequantumdots@ion-imprinted polymer sensing sites of the bottom layer, and the design also improved the portability of the device by transferring the liquid phase of ZnSeQDs@ion imprinted polymers to solid glass fiber paper with good sensitivity, selectivity and multiplexed detection. (Zhu et al. 2014) detected glucose with the help of a treeshaped paper strip, which showed self-calibration on the test strip and assured the uniformity of the micro flows in different branches at the same time, which helped to collect multiple data of the samples without any delay because of the minimum effect of environmental conditions (temperature, humidity, pressure, etc.) on self-calibration. At the same time, a Y-shaped strip can semi-quantitatively judge whether the concentration in blood serum is low level, average level, or high level, and directly provides a result of "Yes or No."

\subsection{Surface modification via nano functionalization}

The inclusive aim of surface modification is to get reliable, quick, and economical testing results, and it is required to develop POCT devices with better performance, usability, and portability. This can be achieved through the surface medication of the electrodes and detection zones by the smart material. These smart materials are designed materials and play a vital role by offering the capability to capture 
and release specific components at different conditions by isolating the analyte during sample pre-treatment (Sow et al. 2020). The electrodes and the detection zone of the $\mu$ PADs modified through the various metals' nanoparticles and bio-polymers in different concentrations. Due to its high surface area, these molecules have an effective surface for bio-molecule immobilization, better confirmation, and superior biological activities resulting in improved sensing characteristics (Gupta et al. 2017).

\subsection{Enhancement through silver nano-particles}

The Ag NPs belong to the most substantial conductive nanomaterial family due to its small molecular diameter, large surface area, and fast charge transfer, it improves the detection capabilities of the device (Prasad et al. 2011; Rycenga et al. 2011). The conductivity of Ag particles increases through the high density of Ag NPs, which can be achieved through small particle and air stability results of Ag NPs; these properties promote the use of Ag NPs as conductive ink in paper-based flexible electronics ( $\mathrm{Li}$ et al. 2014). It is reported that silver nanoplates behave as an extremely sensitive material towards the inorganic anions (Jiang and Yu 2008). (Ding et al. 2020) used silver/silver chloride $(\mathrm{Ag} / \mathrm{AgCl})$ as a reference element for the ion determination in clinically relevant samples and environmental samples. Ferreira et al. (Ferreira et al. 2015) introduced novel optical silver nanoparticles-based sensor for the quantification of ascorbic acid. The sensitivity of the colorimetric measurement of ascorbic acid increases due to the cluster formation growth property of the silver nanoparticles. Ratnarathorn et al. (Ratnarathorn et al. 2012) demonstrated the use of Ag NPs for the colorimetric sensing of copper ion $\left(\mathrm{Cu}^{2+}\right)$ in the paper-based device for the rapid, easy and, economical POCT. The color change was observed through the naked eye due to the tremendously elevated extinction coefficient of the Ag NPs.

\subsection{Enhancement through nano-bio-polymer}

Bio-functionalization is performed for the enhancement of the working substrate. Chitosan is a common bio-functionalized material and its derivatives have been commonly used for the stability of the antibodies due to its cationic properties (Atwe et al. 2014). It also persists in some important properties, which makes it exceptional from other biopolymer. Due to the exceptional properties such as biocompatibility, easy solubilizes in an aqueous medium, bio-degradability, hydrophilicity, etc., chitosan became a great immobilization matrix for the fabrication of a wide range of biosensors (Baranwal et al. 2018; Patel et al. 2015). Further organosilicate polymer composites have also been reported as a protein immobilization platform for bio sensing application (Gupta et al. 2014, 2021). (Parween et al. 2020) evaluated different bio-functionalization methods of chitosan on paper-based schemes for better stability and immobilization of antibodies and found that chitosan-modified schemes show better stability at room temperature. On the other hand, sodium hydroxide cross-linked chitosan (CHI-NaOH) bio-functionalized schemes showed stability for more than 100 days due to the increment in degree of carboxymethylation and deacetyalation of chitosan, which is maximum in comparison to other functionalized methods. Chitosan is a biopolymer that is extensively used to modify the bio sensing surface and improves the capability of the direct electron transfer between a reactive surface and enzyme, by providing a suitable microenvironment. Chitosan has very high surface area supporting cellulose fiber to absorb enzymes and chromogenic substrate (Gabriel et al. 2016; Li et al. 2019).

\subsection{Enhancement through gold nano-particles}

Gold nanoparticles (Au NPs) are unproblematic to be conjugated with toxins, hormones, immunoglobulins, antibiotics and other biological macromolecules through electrostatic interaction without affecting their biological activity. It is biocompatible, carries size tunable properties and provides a range of color code based on the nano particle size. It is reported that decoration of Au NPs on the surface of rGOTEPA, enhanced biocompatibility and stability of the rGOTEPA/Au nano composite which enhanced the acceleration of the electron transfer to the screen printed electrodes (SPEs) as well as capturing antibodies (Cao et al. 2017). Further, it is reported that enzyme-modified Au NPs shows high color intensity and sensitivity in comparison to traditional Au NPs (Parolo et al. 2013). Further, two new optical assay techniques, direct reduction and bienzymatic methods are proposed for the detection of sugars in beverages. The direct reduction of Au NPs provides good sensitivity, while bienzymatic method provides lower cost and simplification, and it does not suffer bleaching due to the high stability of the Au NPs (Palazzo et al. 2012). It is reported that closed Au-bipolar electrodes persist excellent conductivity in comparison of graphite electrodes, a good choice over the three-electrode system, because it can distinguish the anode and cathode into separate compartments, avoid any interference and have high current efficiency. Au-Pd used as a catalyst which could catalyze the reduction of hydrogen peroxide $\left(\mathrm{H}_{2} \mathrm{O}_{2}\right)$ for signal amplification (Wang et al. 2020a). It showed good sensitivity and selectivity. Due to the following properties, it is highly reported nanomaterials. Figure 3 shows a complete cycle representation of ECL biosensor for the detection of miRNA-155. 


\subsection{Enhancement through metal oxide nanoparticles}

The contribution of metal oxide nanoparticles (MONPs) is well known, and therefore it has been heavily explored as heterogeneous catalysis. To prepare and understand the catalytic materials, the sol-gel method is utilized due to its exceptional versatility. MONPs show its good biocompatibility, chemical stability, high surface area, and ability to transfer electrons at a higher speed. These characteristics of MONPs make it perfect for immobilization matrices and transduction platforms. Recent research shows that the modification of electrodes through metal oxides can improve the sensitivity of the electrodes. Nano phase metal oxides with unique optical, electrochemical properties, along with the required surface functionalities and electronic properties, offer fascinating bio-platforms for the interaction of bio-recognition elements with transducers for better signal intensification. (Figueredo et al. 2016) described the surface modification of $\mu$ PAD through $\mathrm{Fe}_{3} \mathrm{O}_{4}$ NPs, MWCNT, and Graphene Oxide for the detection of glucose. The reported procedures solve the problem of enzymatic activity and give an enhanced analytical performance at low concentrations. (Evans et al. 2014) fabricated a $\mu \mathrm{PAD}$ through a $\mathrm{CO}_{2}$ laser engraver, and for the enhancement of analytical sensitivity of the $\mu \mathrm{PAD}$, it was immersed in to the suspension of the APTES and modified silica NPS. These NPs can be conveniently entombed in the cellulose structure, which provides strong support for the immobilization of the enzymes. The reported qualitative analysis of lactate, glutamate, and glucose in artificial urine shows improved color intensity and uniformity. (Li et al. 2015) used a carbon ink working electrode decorated with zinc oxide Nano-wires (ZnO NWs) for the glucose detection with higher sensitivity and lower limit of detection due to high enzyme capturing efficiency of $\mathrm{ZnO}$
NWs and high surface to volume ratio. The synthesis of the $\mathrm{ZnO}$ NWs was carried out by using a hydrothermal route and did not require any light-sensitive electron mediator, which explained enhanced bio-sensing stability, and showed sensitivity up to $8.24 \mu \mathrm{A} \mathrm{mM}^{-1} \mathrm{~cm}^{-2}$. (Sun et al. 2018) used $\mathrm{CeO}_{2}$-Au@glucoseoxidase for signal amplification in electrochemical analyses to detect miR-21. A stepwise fabrication detail is shown in Fig. 4.

\subsection{Enhancement through bi- and trimetallic Nanoparticles}

Bi-metallic nanoparticles of $\mathrm{Au}, \mathrm{Pd}, \mathrm{Ag}, \mathrm{Pt}$ show good intrinsic peroxidase-like activity, improve signal amplification (Shen et al. 2015), and enhanced catalytic activity and selectivity (An et al. 2012). Nano clusters of different metals have discrete energy levels for sub-nanometer-size centers, and it connects the missing link between the particles and metal atoms. They persist many diverse properties, viz., high biocompatibility, easy preparation, good photo stability, and low toxicity (Dehghani et al. 2019; Rahimi-Nasrabadi et al. 2017). (Zhu et al. 2017) used $\mathrm{Cu}_{3}\left(\mathrm{PO}_{4}\right)_{2}$ nano crystals in the detection zone with GOx and horseradish peroxidase (HRP) to detect glucose in blood serum. It exhibited a flower-like structure that preserves the activity and enhances the stability of enzymes, and allows for the co-immobilization of the enzymes. (A. Bagheri pebdeni and M. Hosseini 2020) fabricated a $\mu \mathrm{PAD}$ for the detection of bacterial cells through the colorimetric detection method and resulted that dynamic linear range increases and detection limit decrease for a wide variation of the concentration of S.aureus, while using Bimetallic Nano cluster of Au/Pt. A simple mechanism of fabrication and detection is shown in Fig. 5. (Wu et al. 2016) developed a highly sensitive and specific $\mu$ PAD for the detection of living cancer cells with high reproducibility,
Fig. 3 Cycle representation of ECL biosensor for the detection of miRNA-155 based on a paper-based BPE with immobilization, displacement reaction, cycle of target, the immobilization of S1-AuPd.Reproduced with kind permission (Wang et al. 2020a)

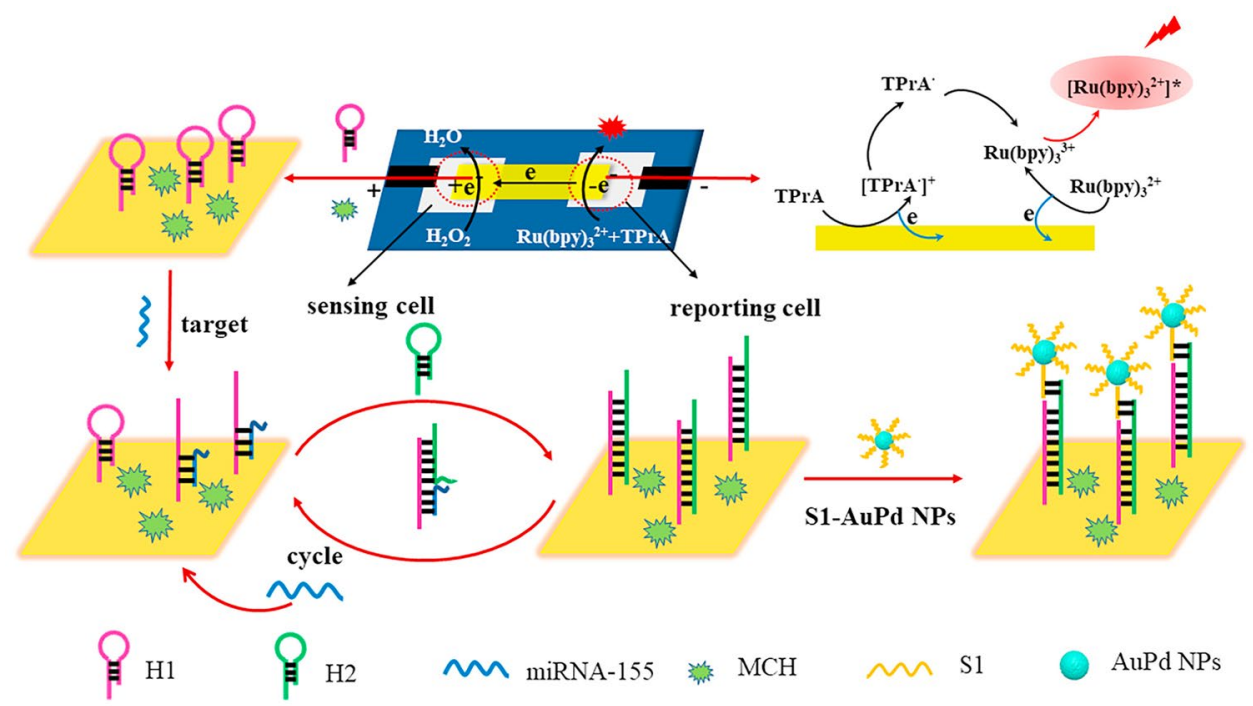


Fig. 4 Illustration of the stepwise biosensor fabrication. Reproduced with kind permission from Sun et al. (2018)

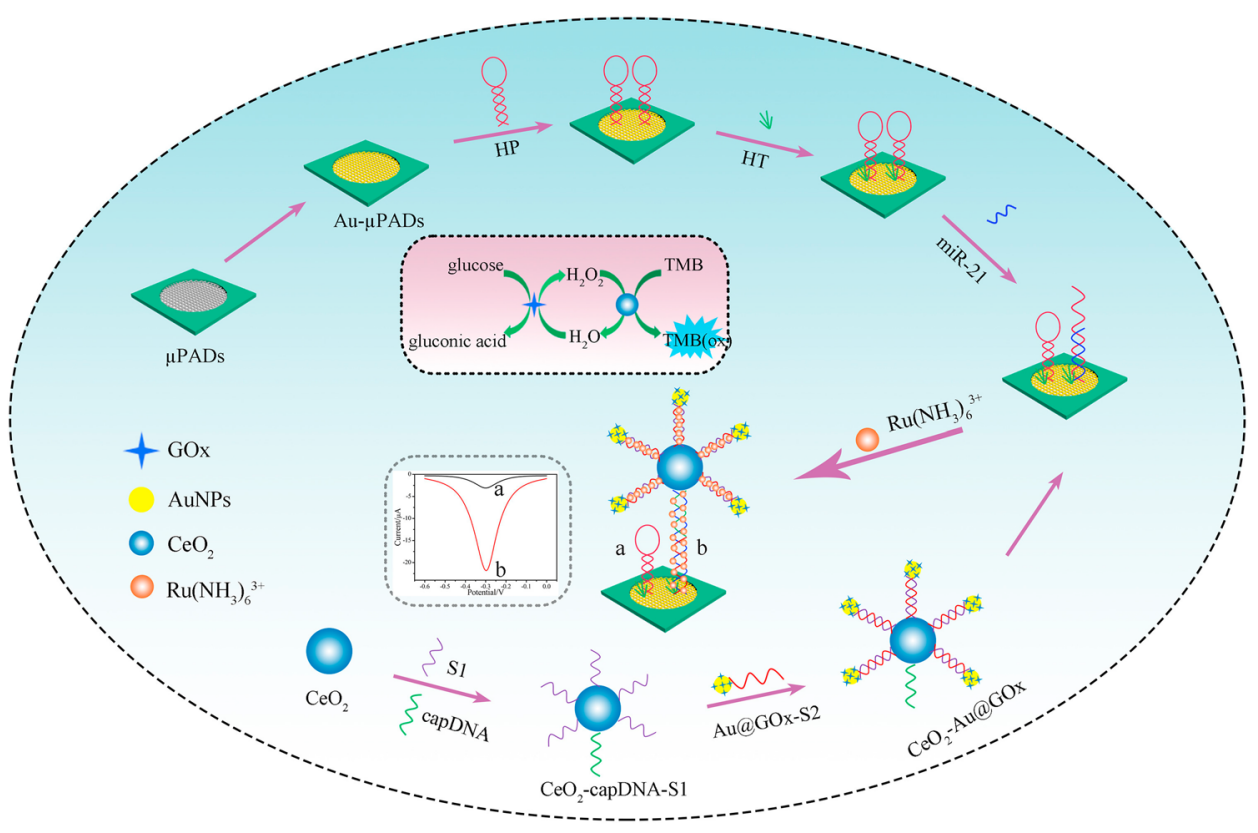

low detection limit, stability, and broad linear range. The paper-based working electrode was developed through the Au@Pd, which shows excellent electrochemical property for cyto-sensing and for the good ECL labeling Pt-Ni alloy used with high loading of carbon dots. (Arshad et al. 2019) developed a simple, selective and sensitive $\mu$ PAD for the detection of explosive nitroaromatics through colorimetric detection technique. The $\mu \mathrm{PAD}$ functionalized through Au@ Ag core-shell nanoparticles with $\beta$-crystamine which shows selective determination of tri-nitro toluene (TNT) due to the charge transfer from electron rich $\beta$-crystamine to TNT.

\subsection{Enhancement through nanoparticles combined with carbon-based nanomaterials}

Graphene is a single layer of carbon atoms closely packed into a 2-D honeycomb structure arrangement. It shows excellent electrical mobility and electrical conductivity for the fabrication of graphene-based electronic material. It has a small band-gap that greatly contributes in electron conduction from target molecules in electrochemical biosensors

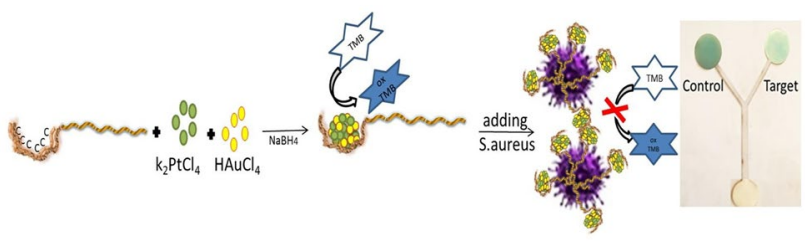

Fig. 5 Simple mechanisms for the formation of $\mathrm{Au} / \mathrm{Pt} \mathrm{NCs}$, and detection of S.aureus through paper. Reproduced with kind permission from A. Bagheri pebdeni and M. Hosseini (2020)
(Mondal et al. 2019; Mondal and Gupta 2018). Graphene can provide ultra-high surface area for the loading of bioreceptorn. It possesses high sensitivity due to low electronic noise from thermal effects. (Deng et al. 2014) synthesized graphene oxide@ $\mathrm{SiO}_{2} @ \mathrm{CeO}_{2}$ hybrid nano sheets (GSCs) through wet chemical strategy as an alternative to the commonly employed peroxidase, and found that these Nano sheets possessed high intrinsic peroxidase activity, and a high potential for the colorimetric indicator. (Fan et al. 2017) used amino-functional graphene, thionine, and gold nanoparticles $\left(\mathrm{NH}_{2}-\mathrm{G} / \mathrm{Thi} / \mathrm{AuNPs}\right)$ for modification of $\mu$ PADs, and found that it showed a wide linear range and low detection limit due to the NH2-G. The NH2-G exhibits excellent biocompatibility and conductivity that accelerate electron transfer and current signal amplification. (Wei et al. 2018) used graphene Nano composite (AuNPs/rGO/THI) coated working electrode for the immobilization of DNA aptamer. The THI was utilized to transduce the biological recognition between DNA aptamer and prostate-specific antigen, which also served as an electrochemical mediator, AuNPs served as an excellent conductor (Au NPs boost the catalytic activity), and rGO facilitates high electron transfer that showed low LOD, and wide linear range. In another work, dopamine was detected by using graphene and sodium dodecyl sulfate surfactant dopamine (Manbohi and Ahmadi 2019). (Liu et al. 2019) used water-dispersible molybdenum oxide quantum dots for highly sensitive and stable sensors to detect $\mathrm{H}_{2} \mathrm{O}_{2}$ released from P12 cells. Molybdenum oxide quantum dots were prepared using a facile ultrasonic-assisted hydrothermal method, and the starting material was $\mathrm{MoS}_{2}$ powder, further synthesized process as shown in the chemical reaction $1,2,3,4$. 


$$
\begin{aligned}
& 2 \mathrm{MoS}_{2}+9 \mathrm{O}_{2}+4 \mathrm{H}_{2} \mathrm{O} \longrightarrow 2 \mathrm{MoO}_{3}+\mathrm{H}_{2} \mathrm{SO}_{4} \\
& \mathrm{H}_{2} \mathrm{SO}_{4}+\mathrm{BaCl}_{2} \longrightarrow \mathrm{BaSO}_{4}+2 \mathrm{HCl} \\
& \mathrm{Na}_{2} \mathrm{CO}_{3}+\mathrm{BaCl}_{2} \longrightarrow \mathrm{BaCO}_{3}+2 \mathrm{NaCl} \\
& \mathrm{BaCO}_{3}+2 \mathrm{HCl} \longrightarrow \mathrm{BaCl}_{2}+\mathrm{CO}_{2}+\mathrm{H}_{2} \mathrm{O}
\end{aligned}
$$

(Zhou 2020) used ZnSe quantum dots (lesser toxic than $\mathrm{CdTe}$ ) for fluorescence detection of cadmium and lead ions. (Cincotto et al. 2019) used graphene quantum dots for direct oxidation of uric acid, whereas graphene quantum dots with creatininase and ruthenium serves as an electrochemical mediator for creatinine oxidation, and the background current increased significantly due to the larger electro active area of graphene quantum dots at the electrode surface.

\subsubsection{Enhancement through up-conversion Nanoparticles (UCNPs)}

The Up-conversion nanoparticles can be defined as a type of luminescent nanomaterials doped with rare earth elements and can convert near-infrared (NIR) excitation light into visible light ultraviolet emitting light (Auzel 2004). It has poor water dispersibility and solubility; it is necessary for chemical modification on the surface. UCNPs persist narrow emission spectrum, low toxicity anti-interference from biological auto fluorescence, and anti-stokes shift compared to quantum dots, which are ideal fluorescent labels in FLFIAs (Tang et al. 2015). (Chen et al. 2017) used a paper supported aptasensor for detection of exosomes (cancer diagnostic marker) by using luminescence resonance energy transfer with up conversion nanoparticles with gold nanoparticles and obtained colored image to quantify the luminescence with a range of $1 \times 10^{4}-1 \times 10^{8}$ particles $/ \mu \mathrm{L}$ and detection limit $1.1 \times 10^{8}$ particles $/ \mu$ L. (Jiang et al. 2017) employed a paper-supported aptasensor for the detection of $\operatorname{IgE}$ in both buffer solution and human serum with a linear range of $0.5-80 \mathrm{ng} / \mathrm{mL}$ by using luminescence resonance energy transfer with carbon nanoparticles as energy acceptor and up conversion nanoparticles used as an energy donor.

\section{Various sensing mechanisms explored in loT assisted paper devices}

The sensing mechanism for $\mu$ PADs is characterized in the following sensing techniques: colorimetric, electrochemical, fluorescent, photo electrochemical, chemiluminescence (CL), electrochemiluminescence (ECL), nanoparticles based sensing, spectrometry sensing (Fu and Wang 2018). Table 2 and Table 3 summarize the recent progresses carried out on different sensing mechanisms used for the diagnosis of health and IoT-enabled paper-based analytical devices, respectively.

\subsection{Colorimetric sensing}

The colorimetric signals are achieved on the paper substrate by the reaction of enzymes and detection substance, and initial color signals can be observed through the uncovered eye, but the intensity of the color signals diagnosed through the image sensors, for instance, charge-coupled device (CCD) or complementary metal-oxide-semiconductor (CMOS) camera with high sensitivity. A simple, rapid, sensitive, and cost-effective hybrid $\mu \mathrm{PAD}$ was developed for the screening of chlorpyrifos-oxon in human serum. A smartphone reader used for getting standardized optical detection and software utilized for extracting RGB intensity (Tsagkaris et al. 2021). (Chen et al. 2012) used N-ethyl-N (3-sulfopropyl)-3-methyl-aniline sodium salt (TOPS)/4AAP in $\mu$ PADs for glucose detection, and DHBS/4AAP for uric acid detection, to enhance the sensitivity of the $\mu$ PADs very effectively, a drying method was employed to reduce the background signal by accelerating the volatilization rate in an incubator resulted in enhanced sensitivity. In another work in this context, Further (Li et al. 2019) reported a double-layered structure combined with $3 \mathrm{D} \mu \mathrm{PAD}$ for the detection of multiple bio-molecules viz. uric acid, glucose, lactate, and choline simultaneously. The detection zones of the $\mu \mathrm{PAD}$ were modified through chitosan, which provides a better microenvironment for the enzyme reactivity as well as better adsorption of the chromogenic substrates and enzymes over cellulose fibers. The reported double-layer structure showed enhanced sensitivity, colorimetric performance, and reproducibility by providing controlled diffusion of colorimetric reagents within a detection zone; further, the detection range and sensitivity increases due to the use of smartphones as a detector. (Jayawardane et al. 2015) proposed a paper (filter paper) based analytical device for the determination of ammonia in wastewater through gas diffusion. This allowed the quantitative conversion of the ammonium ion to molecular ammonia, which diffused across the hydrophobic micro porous Teflon membrane of the device into an adjacent hydrophilic reagent zone containing the acid-base indicator 3-nitrophenol or bromothymol blue. (Tseng et al. 2018) proposed a device based on Jaffe's reaction for performing creatinine detection in the whole blood sample, the reaction zone of $3 \mathrm{D} \mu \mathrm{PAD}$ doped with $\mathrm{NaOH}$ and picric acid reagent Jaffe reaction induced between the reagent and blood plasma creatinine and concentration detected through the colorimetric method. In another work, (Tian et al. 2016) described a novel method for the molecular recognition and amplification in signals by integrating cross-linked target responsive 
Table 2 Paper based health diagnostic devices with various sensing mechanisms

\begin{tabular}{|c|c|c|c|c|c|c|c|c|}
\hline Sr. No & Author (s) & $\begin{array}{l}\text { Fabrication } \\
\text { Method }\end{array}$ & Diagnosis & Method & Enhancement & Sample & Range & LOD \\
\hline 1 & $\begin{array}{l}\text { (Cao et al. } \\
2020)\end{array}$ & $\begin{array}{l}\text { Photolithography } \\
\text { and Screen } \\
\text { Printing }\end{array}$ & Glucose & Electrochemical & $\begin{array}{l}\text { Prussian Blue- } \\
\text { rGO-TEPA/ } \\
\text { PB }\end{array}$ & Blood & $0.1 \mathrm{mM}-25 \mathrm{mM}$ & $25 \mu \mathrm{M}$ \\
\hline 2 & $\begin{array}{l}\text { (Noiphung } \\
\text { et al. 2013) }\end{array}$ & Wax Dipping & Glucose & Electrochemical & PB-SPEs & $\begin{array}{l}\text { Whole } \\
\text { Blood }\end{array}$ & $0-33.1 \mathrm{mM}$ & - \\
\hline 3 & (Li et al. 2015) & Wax Printing & Glucose & Electrochemical & $\mathrm{ZnO}$ NWs & $\begin{array}{r}\text { Human } \\
\text { serum }\end{array}$ & $0-15 \mathrm{mM}$ & $\begin{array}{l}59.5 \\
\mu \mathrm{M}\end{array}$ \\
\hline 4 & $\begin{array}{l}\text { (Chaiyo et al. } \\
\text { 2017) }\end{array}$ & Wax Printing & Glucose & Electrochemical & $\begin{array}{l}\text { CoPC/G/IL/ } \\
\text { SPCE }\end{array}$ & $\begin{array}{l}\text { Honey } \\
\text { White } \\
\text { wine } \\
\text { Human } \\
\text { Serum }\end{array}$ & $\begin{array}{l}0.01-1.3 \mathrm{mM} \\
\text { And } 1.3- \\
5.0 \mathrm{mM}\end{array}$ & $0.67 \mu \mathrm{M}$ \\
\hline 5 & $\begin{array}{l}\text { (Deng et al. } \\
\text { 2014) }\end{array}$ & Photolithography & Glucose & Colorimetric & $\begin{array}{l}\mathrm{GO} @ \mathrm{SiO}_{2} @ \\
\mathrm{CeO}_{2} \\
\text { Nano sheets } \\
\text { (GSCs) }\end{array}$ & $\begin{array}{l}\text { Human } \\
\text { serum } \\
\text { and } \\
\text { urine } \\
\text { samples }\end{array}$ & $0.5-30 \mathrm{mM}$ & $9 \mathrm{nM}$ \\
\hline 6 & $\begin{array}{l}\text { (Figueredo } \\
\text { et al. 2016) }\end{array}$ & $\mathrm{CO}_{2}$ laser cutting & Glucose & Colorimetric & $\begin{array}{l}\text { MNPs } \\
\text { MWCNT } \\
\text { GO }\end{array}$ & $\begin{array}{l}\text { Artificial } \\
\text { Urine }\end{array}$ & $\begin{array}{l}0.05-1 \mathrm{mM} \\
0.05-1 \mathrm{mM} \\
0-1 \mathrm{mM}\end{array}$ & $\begin{array}{l}43 \mu \mathrm{M} \\
62 \mu \mathrm{M} \\
18 \mu \mathrm{M}\end{array}$ \\
\hline 7 & $\begin{array}{l}\text { (Soni and Jha } \\
\text { 2015) }\end{array}$ & - & Glucose & Colorimetric & - & Saliva & $9-1350 \mathrm{mg} / \mathrm{dL}$ & $22.2 \mathrm{mg} / \mathrm{dL}$ \\
\hline 8 & $\begin{array}{l}\text { (Zhu et al. } \\
\text { 2014) }\end{array}$ & $\begin{array}{l}\text { Normal cutting of } \\
\text { chromatography } \\
\text { paper }\end{array}$ & Glucose & Colorimetric & GOD/HRP & Serum & $1-11 \mathrm{mM}$ & $0.3 \mathrm{mM}$ \\
\hline 9 & $\begin{array}{l}\text { (Zhu et al. } \\
\text { 2017) }\end{array}$ & Wax Printing & Glucose & Colorimetric & $\mathrm{Cu}_{3}(\mathrm{PO} 4)_{2}$ & Serum & $0.1-10.0 \mathrm{mM}$ & $25 \mu \mathrm{M}$ \\
\hline 10 & $\begin{array}{l}\text { (Gabriel et al. } \\
\text { 2016) }\end{array}$ & Stamping & $\begin{array}{l}\text { Uric Acid } \\
\text { Glucose }\end{array}$ & Colorimetric & Chitosan & $\begin{array}{l}\text { Artificial } \\
\text { serum/ } \\
\text { human } \\
\text { tear } \\
\text { sample }\end{array}$ & $0.1-1.0 \mathrm{mM}$ & $\begin{array}{l}0.037 \mathrm{mM} \\
0.023 \mathrm{mM}\end{array}$ \\
\hline 11 & $\begin{array}{l}\text { (Chen et al. } \\
\text { 2012) }\end{array}$ & Photolithography & $\begin{array}{l}\text { Uric Acid } \\
\text { Glucose }\end{array}$ & Colorimetric & $\begin{array}{l}\text { Bi-enzyme } \\
\text { (GOD/UAO) }\end{array}$ & $\begin{array}{l}\text { Human } \\
\text { Serum }\end{array}$ & $50 \mu \mathrm{M}-1.0 \mathrm{mM}$ & $\begin{array}{l}43.1 \mu \mathrm{M} \\
38.1 \mu \mathrm{M}\end{array}$ \\
\hline 12 & $\begin{array}{l}\text { (Ruecha et al. } \\
\text { 2014) }\end{array}$ & $\begin{array}{l}\text { Paper-wax print- } \\
\text { ing }\end{array}$ & Cholesterol & Electrochemical & G/PVP/PANI & $\begin{array}{l}\text { Human } \\
\text { Serum }\end{array}$ & $50 \mu \mathrm{M}-10 \mathrm{mM}$ & $1 \mu \mathrm{M}$ \\
\hline \multirow[t]{2}{*}{13} & $\begin{array}{l}\text { (Wang et al. } \\
\text { 2019) }\end{array}$ & $\begin{array}{l}\text { Wax Printing and } \\
\text { Screen Printing }\end{array}$ & CEA & $\begin{array}{l}\text { Label Free Elec- } \\
\text { trochemical }\end{array}$ & $\begin{array}{l}\text { NG-THI-Au } \\
\text { NPs }\end{array}$ & Serum & $\begin{array}{l}0.01-500 \\
\text { ngmL }^{-1}\end{array}$ & $2 \mathrm{pgmL}^{-1}$ \\
\hline & & & NSE & & & & $\begin{array}{c}0.05-500 \\
\text { ngmL }^{-1}\end{array}$ & $10 \mathrm{pgmL}^{-1}$ \\
\hline 14 & $\begin{array}{l}\text { (Fan } \\
\quad \text { et al. 2017) }\end{array}$ & $\begin{array}{l}\text { Wax Printing and } \\
\text { Screen Printing }\end{array}$ & $\begin{array}{l}\text { NSE- } \\
\text { Lung Cancer }\end{array}$ & Electrochemical & $\begin{array}{l}\mathrm{NH}_{2}-\mathrm{G} / \mathrm{Thi} / \\
\text { AuNPs }\end{array}$ & Serum & $\begin{array}{l}1-500 \\
\text { ngmL }^{-1}\end{array}$ & $\begin{array}{l}10 \\
\operatorname{pgmL}^{-1}\end{array}$ \\
\hline 15 & $\begin{array}{l}\text { (Evans et al. } \\
\text { 2014) }\end{array}$ & $\begin{array}{l}\mathrm{CO}_{2} \text { laser } \\
\text { engraver }\end{array}$ & $\begin{array}{l}\text { Lactate } \\
\text { Glucose } \\
\text { Glutamate }\end{array}$ & Colorimetric & $\begin{array}{l}\text { Silica Nanopar- } \\
\text { ticles }\end{array}$ & $\begin{array}{l}\text { Artificial } \\
\text { Urine }\end{array}$ & $\begin{array}{l}0.63-3.75 \mathrm{mM} \\
0.5-10 \mathrm{mM} \\
0.25-7.50 \mathrm{mM}\end{array}$ & $\begin{array}{l}0.63 \mathrm{mM} \\
0.50 \mathrm{mM} \\
0.25 \mathrm{mM}\end{array}$ \\
\hline \multirow[t]{4}{*}{16} & (Li et al. 2019) & $\begin{array}{l}\text { Wax Screen } \\
\text { Printing }\end{array}$ & Glucose & Colorimetric & Chitosan & Serum & $\begin{array}{l}0.01-10 \\
\mathrm{mML}^{-1}\end{array}$ & $3 \mu \mathrm{M}$ \\
\hline & & & Uric acid & & & & $\begin{array}{l}0.01-5.0 \\
\mathrm{mML}^{-1}\end{array}$ & $5 \mu \mathrm{M}$ \\
\hline & & & Lactate & & & & $\begin{array}{c}0.04-10.0 \\
\mathrm{mML}^{-1}\end{array}$ & $30 \mu \mathrm{M}$ \\
\hline & & & Choline & & & & $\begin{array}{c}0.04-24.0 \\
\mathrm{mML}^{-1}\end{array}$ & $10 \mu \mathrm{M}$ \\
\hline
\end{tabular}


Table 2 (continued)

\begin{tabular}{|c|c|c|c|c|c|c|c|c|}
\hline Sr. No & Author (s) & $\begin{array}{l}\text { Fabrication } \\
\text { Method }\end{array}$ & Diagnosis & Method & Enhancement & Sample & Range & LOD \\
\hline 17 & $\begin{array}{l}\text { (Ruiz-Vega } \\
\text { et al. 2019) }\end{array}$ & SPCE & MMP-9 & Electrochemical & $\begin{array}{l}\text { Magnetic } \\
\text { Beads }\end{array}$ & Plasma & $0.03-2 \mathrm{ngmL}^{-1}$ & $\begin{array}{l}0.01 \\
\operatorname{ngmL}^{-1}\end{array}$ \\
\hline 18 & $\begin{array}{l}\text { (Wei et al. } \\
\text { 2018) }\end{array}$ & $\begin{array}{l}\text { Wax Printing and } \\
\text { Screen Printing }\end{array}$ & PSA & Electrochemical & $\begin{array}{l}\text { AuNPs/rGO/ } \\
\text { THI }\end{array}$ & Serum & $\begin{array}{l}0.05-200 \\
\text { ngmL }^{-1}\end{array}$ & $10 \mathrm{pgmL}^{-1}$ \\
\hline 19 & $\begin{array}{l}\text { (Wang, et al. } \\
\text { 2020a) }\end{array}$ & $\begin{array}{l}\text { Wax Printing, } \\
\text { Screen Printing, } \\
\text { In-situ }\end{array}$ & miRNA-155 & ECL & AuPd NPs & & $1 \mathrm{pM}-10 \mu \mathrm{M}$ & $0.67 \mathrm{pM}$ \\
\hline \multirow[t]{3}{*}{20} & $\begin{array}{l}\text { (Jiao et al. } \\
2020)\end{array}$ & Wax Printing & CEA & $\begin{array}{l}\text { Fluorescent } \\
\text { Immunoassay }\end{array}$ & & Serum & $\begin{array}{l}0.1-1000 \\
\text { ngmL }^{-1}\end{array}$ & $0.03 \mathrm{ngmL}^{-1}$ \\
\hline & & & AFP & & & & $\begin{array}{l}0.1-1000 \\
\text { ngmL }^{-1}\end{array}$ & $0.05 \mathrm{ngmL}^{-1}$ \\
\hline & & & CA199 & & & & $\begin{array}{c}0.1-1000 \\
\mathrm{UmL}^{-1}\end{array}$ & $0.09 \mathrm{UmL}^{-1}$ \\
\hline 21 & $\begin{array}{l}\text { (Chen et al. } \\
\text { 2018) }\end{array}$ & SPE & HBsAg & ECL & $\begin{array}{l}\text { Magnetic } \\
\text { Beads }\end{array}$ & Serum & $\begin{array}{l}34.2 \mathrm{pgmL}^{-1}- \\
34.2 \mathrm{ngmL}^{-1}\end{array}$ & $34.2 \mathrm{pgmL}^{1}$ \\
\hline 22 & $\begin{array}{l}\text { (Cao et al. } \\
\text { 2017) }\end{array}$ & Photolithography & AFP & Electrochemical & rGO-TEPA/Au & Serum & $\begin{array}{l}0.01-100.0 \\
\text { ngmL }^{-1}\end{array}$ & $\begin{array}{l}0.005 \\
\text { ngmL }^{-1}\end{array}$ \\
\hline 23 & $\begin{array}{l}\text { (Sun et al. } \\
\text { 2018) }\end{array}$ & -- & miR-21 & Electrochemical & $\mathrm{CeO}_{2}$ & $\begin{array}{l}\text { Human } \\
\text { Serum }\end{array}$ & $1000 \mathrm{fM}$ & $0.434 \mathrm{fM}$ \\
\hline 24 & $\begin{array}{l}\text { (Manbohi } \\
\text { and Ahmadi } \\
\text { 2019) }\end{array}$ & Wax Stamping & Dopamine & Electrochemical & Graphene & $\begin{array}{l}\text { Blood } \\
\text { and } \\
\text { Urine }\end{array}$ & $\begin{array}{l}0.5-120 \\
\mu \mathrm{M}\end{array}$ & $0.01 \mu \mathrm{M}$ \\
\hline 25 & $\begin{array}{l}\text { (Cao et al. Jun. } \\
\text { 2017) }\end{array}$ & $\begin{array}{l}\text { Photolithography } \\
\text { and Screen } \\
\text {-printing tech- } \\
\text { nology }\end{array}$ & HCG & Electrochemical & GNPs & Serum & $\begin{array}{l}\text { 1.0mIUmL } \\
-100.0 \\
\text { IUmL }^{-1}\end{array}$ & $\begin{array}{l}0.36 \\
\mathrm{mIUmL}^{-1}\end{array}$ \\
\hline 26 & $\begin{array}{l}\text { (Wang et al. } \\
\text { 2016) }\end{array}$ & $\begin{array}{l}\text { Wax Printing and } \\
\text { Screen Printing }\end{array}$ & CEA & Electrochemical & $\begin{array}{l}\mathrm{NH}_{2}-\mathrm{G} / \mathrm{Thi} / \\
\text { AuNPs }\end{array}$ & Serum & $\begin{array}{c}0.05-500 \\
\text { ngmL }^{-1}\end{array}$ & $10 \mathrm{pgmL}^{-1}$ \\
\hline 27 & $\begin{array}{l}\text { (Cincotto et al. } \\
\text { 2019) }\end{array}$ & Craft Printing & $\begin{array}{l}\text { Uric Acid } \\
\text { Creatininase }\end{array}$ & Electrochemical & $\begin{array}{l}\text { Graphene } \\
\text { Quantum } \\
\text { Dots }\end{array}$ & $\begin{array}{l}\text { Human } \\
\text { Serum }\end{array}$ & $\begin{array}{c}0.010-3.0 \\
\mu \mathrm{mL}^{-1}\end{array}$ & $\begin{array}{l}8.4 \mathrm{nmL}^{-1} \\
3.7 \mathrm{nmL}^{-1}\end{array}$ \\
\hline 28 & $\begin{array}{l}\text { (Zamora- } \\
\text { Gálvez et al. } \\
\text { 2018) }\end{array}$ & Printing & $\operatorname{IgG}$ & Fluorescence & QDs-GO & Serum & $\begin{array}{l}1-1000 \\
\text { ngmL }^{-1}\end{array}$ & $6.30 \mathrm{ngmL}^{-1}$ \\
\hline 29 & $\begin{array}{l}\text { (Anjana et al. } \\
\text { 2018) }\end{array}$ & - & Bilirubin & Fluorescence & $\mathrm{S}, \mathrm{N}-\mathrm{CDs}$ & Serum & $0.2-2.0 \mathrm{nM}$ & $0.12 \mathrm{nM}$ \\
\hline 30 & $\begin{array}{l}\text { (Ruiz-Vega } \\
\quad \text { et al. 2020) }\end{array}$ & SPCE & Malaria(pfLDH) & Electrochemical & $\begin{array}{l}\text { Magnetic } \\
\text { Beads }\end{array}$ & $\begin{array}{l}\text { Whole } \\
\text { blood } \\
\text { sample }\end{array}$ & $\begin{array}{r}0.006-1.5 \% \text { in } \\
\text { culture } \mathrm{RBC}\end{array}$ & $200 \mathrm{ngmL}^{-1}$ \\
\hline
\end{tabular}

hydrogel (glucoamylase-trapped aptamer) with the cascaded enzymatic reaction for the $\mu \mathrm{PAD}$ POCT. The proposed device tested for the urine and cocaine in the buffer. (Son et al. 2018) developed a polydiacetylene-based paper sensor for naked-eye detection of pandemic influenza a (pH1N1) virus. It (PDA-paper chips) changes color under various external conditions (temperature, $\mathrm{pH}$ ) form blue to red. Further, an app was developed for the detection of viruses at low concentrations. (Im et al. 2016) developed an optical biosensor for the detection of glucose and lactate as a biomarker for monitoring of cell growth by using a smart-phone with high accuracy and reproducibility with linear range $0.3-8.0 \mathrm{mM}$ and LOD $0.3 \mathrm{mM}$ for glucose detection and linear range 0.02
$-0.50 \mathrm{mM}$ and LOD $0.02 \mathrm{mM}$ for lactate detection as shown in Fig. 6. (Chun et al. 2014) developed a $\mu$ PAD containing both enzymes mediated assay function and sample transporting function for easy and accurate glucose bio sensing and used smartphones as a potential signal transducer. ( $\mathrm{Li}$ et al. 2018) constructed a $\mu$ PAD through the direct synthesis of enzyme-inorganic hybrid nanomaterial's on the paper matrix, for the in situ growth of GOx@ $\mathrm{Mn}_{3}\left(\mathrm{PO}_{4}\right)_{2}$ hybrid function materials in an inorganic manner solution $\left(\mathrm{MnSO}_{4}\right.$ and $\mathrm{KH}_{2} \mathrm{PO}_{4}$ ) containing a diluted enzyme glucose oxidase and GOx pipette on to cellulose paper. The centrifugation and the dry process eliminated through this new approach of the in-situ growth of an enzyme-inorganic hybrid on a 
Table 3 IoT-enabled POCT devices for various diagnoses reported in the last 5 years

\begin{tabular}{|c|c|c|c|c|c|c|}
\hline S. No & Author & Fabrication Method & Diagnostic & Method & LOD & Data acquisition and analysis \\
\hline 1 & $\begin{array}{l}\text { (Liu et al. } \\
\text { 2020) }\end{array}$ & Paper substrate & Nucleic acid & Fluorescence & $2 * 10^{3}$ copies $/ \mu \mathrm{L}$ & $\begin{array}{l}\text { IoT microprocessor for data col- } \\
\text { lection cloud storage. }\end{array}$ \\
\hline 2 & $\begin{array}{l}\text { (Chen } \\
\text { et al. 2016) }\end{array}$ & $\begin{array}{l}\text { Wax screen print- } \\
\text { ing }\end{array}$ & Glucose & Electrochemiluminescence & $17 \mu \mathrm{M}$ & $\begin{array}{l}\text { Smartphone imaging, wireless } \\
\text { transmission of ECL signal to } \\
\text { PC. }\end{array}$ \\
\hline 3 & $\begin{array}{l}\text { (Tsagkaris et al. } \\
\text { 2021) }\end{array}$ & Wax printing & $\begin{array}{l}\text { Chlorpyrifos- } \\
\text { oxon }\end{array}$ & Colorimetric & $0.033 \mu \mathrm{g} \mathrm{L}^{-1}$ & $\begin{array}{l}\text { Smartphone reader for optical } \\
\text { detection, a smartphone app for } \\
\text { results display. }\end{array}$ \\
\hline 4 & (Im et al. 2016) & Wax printing & $\begin{array}{l}\text { Glucose and } \\
\text { lactate }\end{array}$ & Colorimetric & 0.3 and $0.02 \mathrm{mM}$ & $\begin{array}{l}\text { Smartphone as optical sensing } \\
\text { system, color intensity quantifi- } \\
\text { cation through software. }\end{array}$ \\
\hline 5 & $\begin{array}{l}\text { (Calabria et al. } \\
\text { 2017) }\end{array}$ & Layer by layer & $\begin{array}{r}\text { Salivary } \\
\text { lactate }\end{array}$ & Colorimetric & $0.1 \mathrm{mML}^{-1}$ & $\begin{array}{l}\text { Smartphone for detection, com- } \\
\text { putational online data analysis } \\
\text { for data transfer. }\end{array}$ \\
\hline 6 & $\begin{array}{l}\text { (Kaarj et al. } \\
\text { 2018) }\end{array}$ & Wax printing & Zika Virus & Fluorescence & 1 copie $/ \mu \mathrm{L}$ & $\begin{array}{l}\text { Smartphone for image quantifica- } \\
\text { tion and data analysis. }\end{array}$ \\
\hline 7 & $\begin{array}{l}\text { (Monisha } \\
\text { et al. 2021) }\end{array}$ & Inkjet printing & $\mathrm{Hg}^{+2}$ & Colorimetric & $10 \mu \mathrm{g} \mathrm{L}^{-1}$ & $\begin{array}{l}\text { Smartphone for image quantifica- } \\
\text { tion and data analysis. }\end{array}$ \\
\hline 8 & $\begin{array}{l}\text { (Franco } \\
\text { et al. 2021) }\end{array}$ & Wax Printing & $\mathrm{Cu}^{2+}$ & Colorimetric & $0.034 \mathrm{mgL}^{-1}$ & $\begin{array}{l}\text { Smartphone for image quantifica- } \\
\text { tion and data analysis. }\end{array}$ \\
\hline 9 & $\begin{array}{l}\text { (Ulep et al. } \\
\text { 2020) }\end{array}$ & Wax Printing & $\begin{array}{l}\text { ROR } 1+\text { can- } \\
\text { cer cells }\end{array}$ & Fluorescence & 0.1 cells $/ \mu \mathrm{L}$ & $\begin{array}{l}\text { Smartphone for fluorescence } \\
\text { microscopy and image process- } \\
\text { ing and software for analysis. }\end{array}$ \\
\hline 10 & $\begin{array}{l}\text { (Kou et al. } \\
\text { 2020) }\end{array}$ & Cut and stack & $\begin{array}{l}\text { Glucose and } \\
\text { uric acid }\end{array}$ & Colorimetric & - & $\begin{array}{l}\text { Smartphone for image quantifica- } \\
\text { tion and data analysis. }\end{array}$ \\
\hline
\end{tabular}

paper matrix. (Calabria et al. 2017) developed a smartphone biosensor in which reagents are necessary for analysis coentrapped on paper in a wafer-like bilayer film of polyelectrolytes to detect L-lactate in oral fluid with $0.1 \mathrm{mML}^{-1}$ limit of detection. A simple, fast and sensitive approach for detecting Zika virus executed with a smartphone and $\mu$ PADs, an image of the detection zone is captured through the smartphone camera for real-time quantification. The color intensities are evaluated through the software (Kaarj et al. 2018). In another work, smart-phone is used for the colorimetric detection of $\mathrm{Hg}^{2+}$ in an inkjet-printed paper-based sensor modified with silver nanoparticles. The quantitative analysis performed through calculating intensity of Ag NPs through color detector app and smart-phone (Monisha et al. 2021). In another work, $\mathrm{Cu}^{2+}$ ion presence was determined in sugar cane spirits through the smart-phone camera, by functionalizing paperbased device by the cuprizone. The proposed method is simple, precise and fast in terms of qualitative and quantitative determination (M. de et al. 2021).

\subsection{Electrochemical sensing}

Electrochemical biosensors can be fabricated in different ways, including microfluidic paper-based analytical devices ( $\mu$ PADs), microelectrode array (MEA), and single large electrodes. The most common electrochemical detection methods include cyclic voltammetry, chronoamperometry, and differential pulse voltammetry (DPV) measurements. DPV possesses higher detection precision than chronoamperometry and cyclic voltammetry by reducing background current (Wiley". 2020) and can be used for detection of different POCT Hemoglobin (Hussain et al. 2017), Glucose, etc. in food and beverages. (Cao et al. 2020) constructed a novel 3D microfluidic electrochemical glucose biosensor, where reduced graphene oxide tetraethylenepentamine (rGO-TEPA/PB) was used as a working electrode. The prepared working electrodes showed high conductivity and good electro catalytic reduction towards $\mathrm{H}_{2} \mathrm{O}_{2}$ due to the large specific area. The prepared biosensor showed a broad linear range and low detection limit while detecting glucose in sweat and blood. In another work, (Cao et al. Jun. 2017) designed and constructed a novel $\mu$ PAD for the determination of human chorionic gonadotropin (HCG). Immuno-filtration techniques were introduced for the fabrication of the device, and periodate oxidation was used for the covalent conjugation of the antibodies on the hydrophilic zone of the SPEs. This showed pleasing specificity and sensitivity. (Määttänen et al. 2013) developed a low-cost paper-based platform for electrochemical analyses. Working and counter electrodes directly printed gold stripes and 
Fig. 6 illustrates (1) smart phone-based device with assays of glucose and lactate based on reaction time Reproduced with kind permission from Im et al. (2016), (2) Illustrate main component of the portable detection system and detection box with the effect of reaction temperature (a) and reaction time (b) on the green + blue intensity Reproduced with kind permission from Tseng et al. (2018).
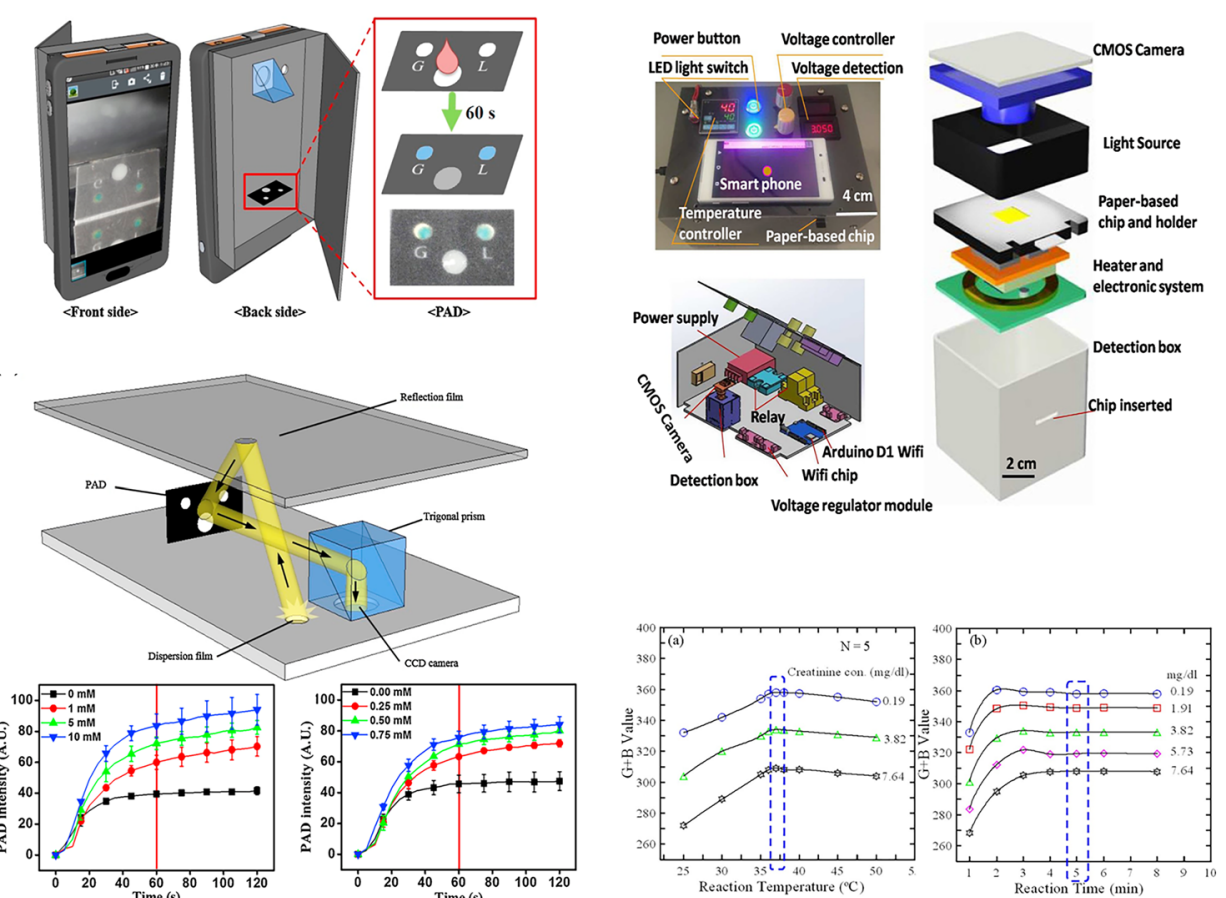

(2) reference electrodes printed with silver stripes onto which silver chloride layer was deposited electrochemically showed performance equal to the conventional electrode with good $\mathrm{pH}$ sensitivity even after five weeks of storage. (Ruecha et al. 2014) prepared a nano composite of graphene / polyvinylpyrrolidone / polyaniline (G/PVP/PANI) and used it for modification of paper-based biosensors via electro-spraying. Adding a small amount of PVP, improved the dispersion of graphene in Nano composites, which increased electrode conductivity and sensitivity of the biosensor; prepared biosensors provide a cost-effective, portable, and disposable paper-based device. (Wang et al. 2019) detected carcinoembryonic antigen (CEA) and NSE for identifying lung cancer by fabricating a paper-based device via wax printing and screen printing with functions of sample auto-injection and sample filtration with the help of NG-THI-AuNPs and prussian blue- poly(3,4-ethylenedioxythiophene)-gold nanoparticles (PB-PEDOT-AuNP), further synthesis of PB-PEDOTAuNPs Nano composite explained through the chemical reaction 5 and 6 .

$$
\begin{aligned}
& \mathrm{Fe}^{3+}+\mathrm{EDOT} \longrightarrow \mathrm{Fe}^{2+}+\text { PEDOT } \\
& \mathrm{Fe}^{2+}+\left[\mathrm{Fe}(\mathrm{CN})_{6}\right]^{3-} \longrightarrow \mathrm{Fe}_{3}\left[\mathrm{Fe}(\mathrm{CN})_{6}\right]_{2}(\mathrm{~PB})
\end{aligned}
$$

The modified electrode worked for promoting the electrode transfer rate as well as immobilization of the CEA, and NSE aptamers. (Noiphung et al. 2013) used Prussian blue modified electrode to detect glucose in whole blood samples using dumbbell-shaped $\mu$ PADs. (Ruiz-Vega et al. 2019) used magnetic beads to develop electrochemical magnetoimmunosensor that performed electrochemical detection and washing on the chip with minimum intervention of the user to detect matrix metalloproteinase 9 (MMP-9). It stated that it was a single step magneto immunoassay for MMP-9 carried out through 8 screens printed electrode and a movable multiplexed fluidic module. The electrochemical detection, magnetic bead confinement under the static condition and reagent adsorption, magnetic bead washing underflow condition provided by the paper device, showed fast simple, and sensitive assay formats. (Wei et al. 2018) used graphene Nano composite for the detection of PSA. The modified electrode (AuNPs/rGO/THI) showed excellent conductivity and gave a specific peak current associated with an electrochemical mediator of THI. (Sun et al. 2018) detected miR-21through synthesized Au Nano rods by In-situ growth methods in $\mu$ PADs for the enhancement of conductivity and catalyzed glucose further electro-catalyzed by $\mathrm{CeO}_{2}$ and gives a wide linear range and low detection limit. (Manbohi and Ahmadi 2019) detected dopamine in blood and urine samples to enhance the sensitivity and selectivity of $\mu \mathrm{PAD}$ toward dopamine. MWCNT, graphene, and $\mathrm{Fe}_{2} \mathrm{O}_{4}$ nanoparticles were utilized, and sodium dodecyl sulfate (SDS) were selected for efficient detection of dopamine. (Wang et al. 2016) developed a highly sensitive label-free $\mu$ PAD for detecting CEA using $\left(\mathrm{NH}_{2}-\mathrm{G}\right) / \mathrm{Thi} /$ $\mathrm{Au}$ NPs Nano composites (synthesized and coated on the screen-printed electrode) to enhance detection sensitivity. 
(Cincotto et al. 2019) developed a device for detecting two analytes at two different spots with the help of one working electrode, through surface modification with graphene quantum dots (QDs) for uric acid oxidation, whereas graphene QDs, creatininase enzyme, and a ruthenium electrochemical mediator for creatinine oxidation. It showed a low detection limit $8.4 \mathrm{nmL}^{-1}$ for uric acid and $3.7 \mathrm{nmL}^{-1}$ for creatinine with high sensitivity, selectivity, and reproducibility. The electro catalytic mechanism at the surface of electrode can be described as chemical reaction 7,8 , and 9 .

$$
\begin{aligned}
& \text { Creatinine }+\mathrm{H}_{2} \mathrm{O} \stackrel{\text { creatininase }}{\longrightarrow} \text { creatine } \\
& \mathrm{Ru}(\mathrm{III})+\text { creatine } \longrightarrow \mathrm{Ru}(\mathrm{II})+\text { products }
\end{aligned}
$$

$$
\mathrm{Ru}(\mathrm{II}) \rightarrow \mathrm{Ru}(\mathrm{III})+\mathrm{e}^{-} \text {(electrochemical reaction) (9) }
$$

(Chaiyo et al. 2017) introduced a novel $\mu$ PAD by modifying a screen-printed carbon electrode with cobalt phthalocyanine, graphene, and an ionic liquid for the non-enzymatic detection of glucose that showed fast electron transfer kinetics and excellent conductivity. It comprised a detection limit of $0.67 \mu \mathrm{M}$ and a wide linear range of $0.01-1.3 \mathrm{mM}$ and $1.3-5.0 \mathrm{mM}$ for glucose. The electro catalytic process can be expressed as chemical reactions 10,11 , and 12 .

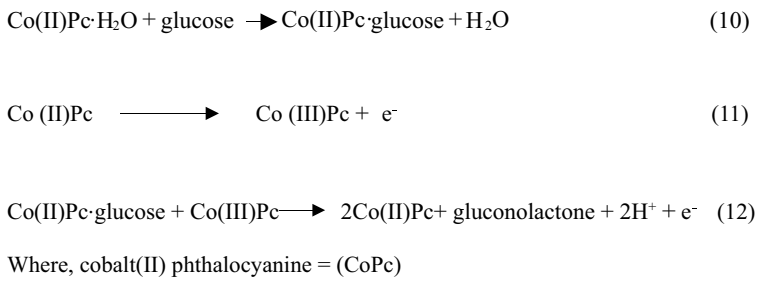

(Adkins and Henry 2015) used microwire electrode as a substitute for the screen-printed electrode for high sensitivity, low resistance, and increase in current density relative to carbon electrode and detected glucose, fructose, and sucrose by using a $\mathrm{Cu}$ electrode in an alkaline solution with a limit of detection $270 \mathrm{nM}, 340 \mathrm{nM}$, and $430 \mathrm{nM}$ respectively. (Ruiz-Vega et al. 2020) developed a paper-based doublesided screen-printed electrode for the detection of malaria Plasmodium falciparum lactate dehydrogenase (pfLDH) through a magnetic bead and found that $\mu$ PADs could be exploited to simplify magneto-immunoassay handling, taking magnetic beads closer to the testing required. (Xu et al. 2020) demonstrated a POCT system which includes handheld electrochemical analyzer and smartphone, for the detection of heavy metals $\left(\mathrm{Cd}^{2+}, \mathrm{Pb}^{+2}, \mathrm{Cu}^{+2}, \mathrm{Hg}^{+2}\right)$ with high sensitivity, Installed APP in the smartphone simultaneously controlled the analyzer as well as receive data and further do the action for the measurements and plotting the graphs of the results. (Fan et al. 2017) reported a wireless
POCT device with a $\mu$ PADs system to detect neuron-specific enolase (NSE) through an electrochemical measurement system to enhance the sensitivity modification done with Nano composites of $\mathrm{NH}_{2}-\mathrm{G} / \mathrm{Thi} / \mathrm{AuNPs}$. The detected results are stored in EEPROM memory automatically. Simultaneously results displayed in android based smartphone via Bluetooth in real-time. Figure 7 illustrates a systematic representation of IoT based POCT system.

\subsection{Fluorescence sensing}

Fluorescence detection is generally performed in a closed light environment at a definite wavelength. The enclosed space provides stability, a closed light environment, and numerous excitation sources with different specific wavelengths, which makes it more demanding compared to the colorimetric detection technique. Fluorescence-based sensing has been extensively utilized in the detection of various biological entities (Gupta et al. 2019; Nayak et al. 2013). (Jiao et al. 2020) constructed a vertical-flow paper-based device for detecting three cancer biomarkers CEA, AFP, and cancer antigen (CA199), for rapid detection within $5 \mathrm{~min}$ with low sample volume and immune response time, fluorescence image captured through the mobile phone under an ultraviolet lamp. (Zhou 2020) used 3D rotary $\mu$ PAD for the detection of $\mathrm{Cd}^{+2}$ and $\mathrm{Pb}^{+2}$ by using a novel fluorescent ZnSe QDs with ion imprinting technology; it enabled them to realize specific and multi-channel determination of the ions. (Guzman et al. 2018) developed an integrated platform consisting of $\mu \mathrm{PAD}$ and a portable detection system for detection of low concentration $\mathrm{CH}_{2} \mathrm{O}$ through Hantzsch reaction (Eq. 13).

$\mathrm{HCHO}+\mathrm{AA}+2$ (AAA) $\longrightarrow$ Dihydropyridine derivative (13)

Between ammonium acetate (AA) and acetoacetanilide (AAA) reagent and formaldehyde, the AA/AAA indicator coated on reaction zone of $\mu \mathrm{PAD}$ and resultant fluorescent dihydropyridine derivative observed through CMOS camera with a detection limit $0.2 \mathrm{ppm}$, Fig. 8 represents a complete detection set-up of low concentration formaldehyde and IoT based LAMP detetor. (Ueland et al. 2016) developed a novel $\mu \mathrm{PAD}$ to extract, filter, and pre-concentrate explosives from the soil for direct analysis; it involves fluorescence quenching based detection. (Liu et al. 2020) fabricated a paper-based loop-mediated isothermal amplification (LAMP) sensing chip for the immediate detections of multiple target genes through different pathogenic bacteria, and analysis is done through the IoT for POCT application. The POCT system consists paper sensor and a portable instrument built on IoT platform. The paper sensor provides the functionality of reagent storage, transportation of samples, 
Fig. 7 (a) illustrates the systematic representation of wireless POCT system with $\mu$ PAD, electrochemical system, and smart phone with customized app operation (Fan et al. 2017), while figure (b) illustrates a systematic layout of glucose detection through $\mu$ PAD with IOT based analysis (Noiphung et al. 2013). \{Reproduced with kind permission\}

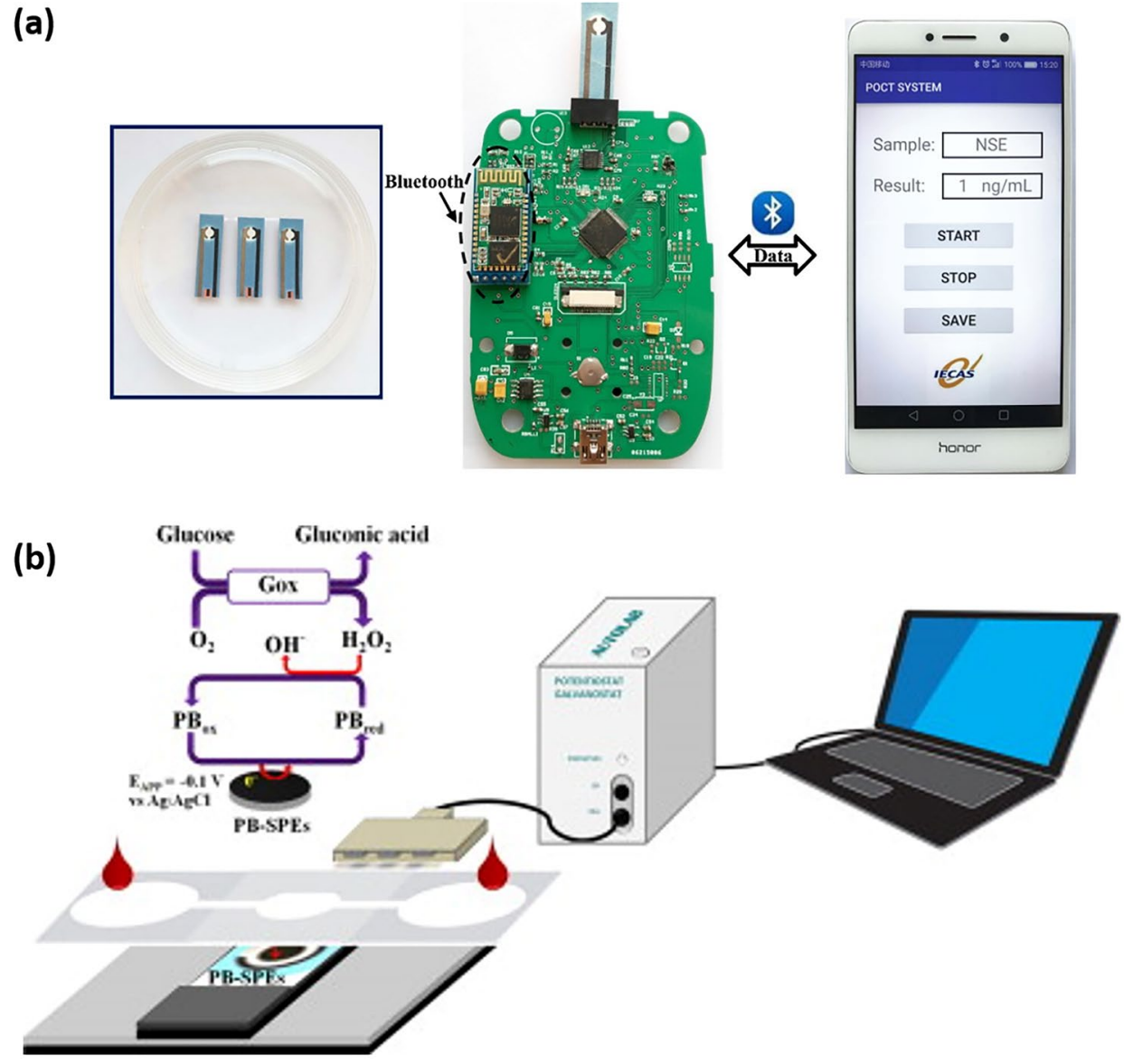

and amplification of nucleic acid. While IoT instrument uses an Arduino microcontroller as fluorescence image collector, temperature controller, and data storage in the cloud through a Wi-Fi network. In another work, a dual layer $\mu$ PAD developed for the detection of $\mathrm{ROR}^{1+}$ cancer cells through wholecell captured imaging and antigen/cell fragment. Where flow fluorescence imaging and velocity analysis done through the smart phone camera (Ulep et al. 2020). Further, (Yetisen et al. 2017) demonstrated a paper-based $\mu$ PAD scheme for the quantitative detection of electrolytes in tears for the early finding of the ocular disorders. Functionalization of detection region is performed through the fluorescent crown ethers, which is sensitive for the mono- and divalent electrolytes. Herein smartphone was utilized as the readout device for the fluorescent outcomes. (Zamora-Gálvez et al. 2018) used a novel design which is based on lateral flow technology in combination with QDs and the usage of graphene oxide for highly sensitive protein detection(IgG), to enhance the sensitivity of fluorescence $\mu \mathrm{PAD}$ assays QDs printed on paper which successfully showed the limit of detection $1.35 \mathrm{ngmL}^{-1}$ for standard buffers and $6.30 \mathrm{ngmL}^{-1}$ for human serums. (Anjana et al. 2018) used carbon dots doped with sulfur and nitrogen prepared via microwave technique to detect bilirubin in human serum with a detection limit of $0.12 \mathrm{nM}$. Citric acid and L-cysteine used for the source of carbon and nitrogen, sulfur, respectively. (Sutariya et al. 2019) used aminopyrine linked calyx[4]arene for highly sensitive and selective detection of $\mathrm{As}^{3+}, \mathrm{Nd}^{3+}$, and $\mathrm{Br}^{-}$. In another work, (Chen et al. 2020) reported a novel naphthofluorescein- based probe $\mathrm{ZN}-2$, which persist longwavelength better sensitivity and faster response in comparison to fluorescein-based probe ZN1. This paper-based system showed the great potential of POCT system for detecting analytes in real samples.

\subsection{Chemiluminescence $(\mathrm{CL})$ sensing}

It is an outstanding recognition method due to its low cost, wide calibration range, high sensitivity, low cost, simple instrumentation, and low background. In the generalized form, signals of CL are generated through the mixing of $\mathrm{CL}$ reagent with the oxidizing reagent in the presence of a catalyst (horseradish and metal ions). (Li et al. 2019) fabricated a double-layer 3D $\mu$ PAD with high resolution temporally resolved chemiluminescence emissions for multiplexed detection of glucose, cholesterol, lactate, and choline, the detection zone modified with the cobalt ion and different oxidase and obtained linear range for cholesterol (0.01-0.4 
Fig. 8 Part (1) shows a schematic design illustrating the housing, temperature management component, microcontroller, and fluorescence detection optics of an IoT-based LAMP detector(Liu et al. 2020), Part (2) represents setup for detection of low concentration formaldehyde (a) portable detection set-up (b) components used in detection box (Guzman et al. 2018). \{Reproduced with kind permission $\}$

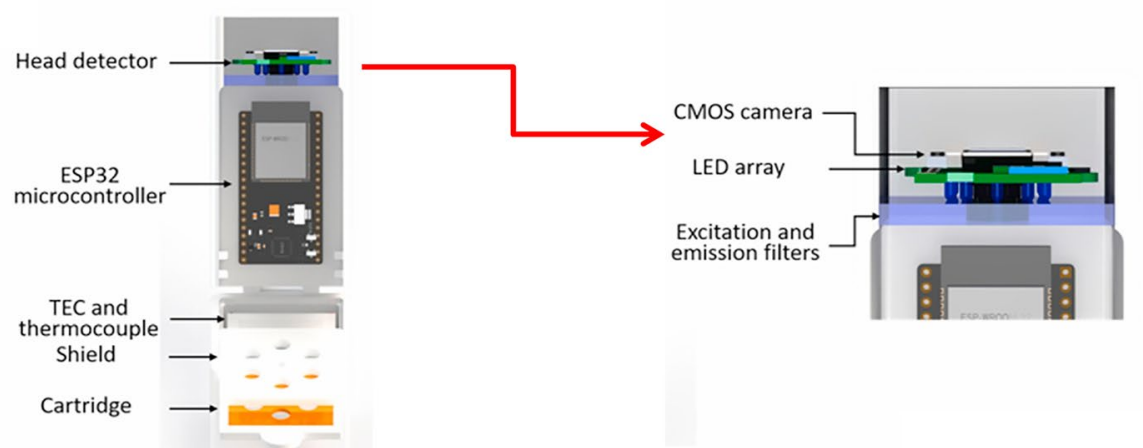

(1)

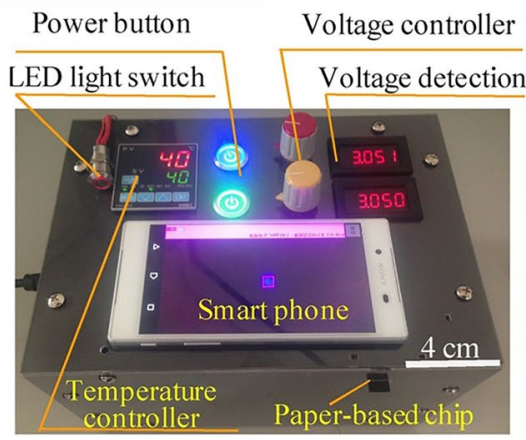

(a)

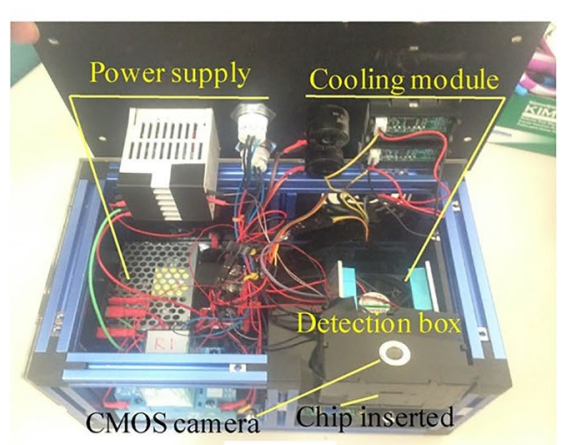

(b)
(2)

$\left.\mathrm{mML}^{-1}\right)$, glucose $\left(0.01-1.0 \mathrm{mML}^{-1}\right)$, lactate $(0.02-5.0$ $\left.\mathrm{mML}^{-1}\right)$, and choline $\left(0.001-1.0 \mathrm{mML}^{-1}\right)$ with detection limit $8,15,6,0.07 \mu \mathrm{ML}^{-1}$ for glucose, lactate, choline and cholesterol respectively. (Liu et al. 2015) used a novel molecularly imprinted polymer $\mu$ PAD to detect dichlorvos, the molecularly imprinted polymer (MIP) layer synthesized and adsorbed on the paper for imprinting of dichlorvos. (Liu et al. 2014) detected dichlorvos (DDV) through paper chromatography; development of DDV was good on paper and separated through the water-soluble vitamins and metal ions. (Li et al. 2020) simultaneously detected three biomarkers (H-FABP, copeptin, and cTnI) through CL detection method in one sample. Simple 3-D sandwich-type CL immunoassays constructed with different detection zone for individual detection of the biomarkers. The amplified signals were obtained through the dual-signal amplification technique by employing $\mathrm{Au}$ NPs and $\mathrm{Co}(\mathrm{II})-\mathrm{Ab}_{2}$-luminol-Au NPs for primary and secondary antibodies, respectively.

\subsection{Electrochemiluminescence sensing}

ECL has an advantage over CL, electrochemistry, and fluorescence in ultrasensitive detection and testing using aptamer, nucleic acids, imprinted polymers, and affinitybased sensing elements. Further advances are happening in the field of signal enhancement and forming integrated systems (Chinnadayyala et al. 2019). (Delaney et al. 2011) described the first approach of electrochemiluminescence at the paper microfluidics combined with screen printed electrodes to detect 2-(dibutylamino) ethanol (DBAE) and nicotinamide adenine dinucleotide (NADH) to the level of $0.9 \mu \mathrm{M}$ and $72 \mu \mathrm{M}$. Further, the mechanism involved for the making of ECL by using $\mathrm{Ru}(\mathrm{bpy})_{3}{ }^{2+}$ and DBAE is projected through the chemical reaction $14,15,16,17,18$.

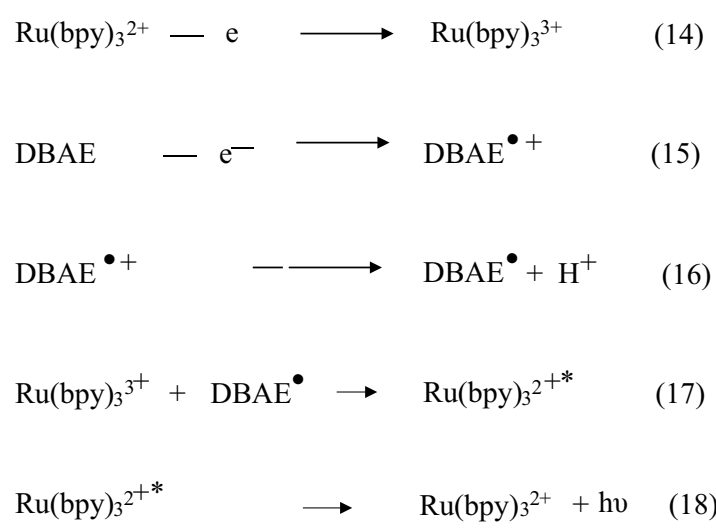

(Wang et al. 2020a) used closed Au-bipolar electrode(BPE) for sensitive detection of miRNA-155 and hybridization chain reaction used for the modification of cathode of a bipolar electrode with DNA 
(S1)-AuPd nanoparticles, which catalyzed $\mathrm{H}_{2} \mathrm{O}_{2}$ reduction and (Ru(bpy) $\left.{ }_{3}{ }^{2+} / \mathrm{TPrA}\right)$ used as an anode of the bipolar electrode. It stated that closed Au-bipolar electrodes persist excellent conductivity in comparison to graphite electrodes, a right choice over the three-electrode system because it can distinguish the anode and cathode into separate compartments, avoid any interference, and have high current efficiency. AuPd used as a catalyst which could catalyze the reduction of $\mathrm{H}_{2} \mathrm{O}_{2}$ for signal amplification. (Chen et al. 2018) developed ECL bio sensing platform for detecting hepatitis B surface antigen (HBsAg) with a modified magnetic suspension sandwich immunoassay method that gave high sensitivity, reliability, and stability. (Chen et al. 2016) developed a handheld paper-based bipolar electrode- ECL system for detecting glucose in phosphate buffer solution and artificial urine with high sensitivity, stability, and reproducibility through a compact device containing a lithium battery for power supply and smartphone for the signal readout shown in Fig. 9. (Liu et al. 2018) prepared a novel paper fluidic crossing channel closed- bipolar electrode in which multiple band-shaped closed-bipolar electrodes situated perpendicular to two parallel channels and multiples detection detected at reporting channel, and analyzed linear glucose range up to $0.08-5 \mathrm{mM}$ and LOD $0.03 \mathrm{mM}$, as well as device, showed duplex detection potential for glucose and uric acid, for the particular process reaction mechanism can be described through chemical reaction 19, 20, 21, 22, 23 .

$\alpha$-D-Glucose $+\mathrm{O}_{2} \stackrel{\text { GOD }}{\longrightarrow}$ gluconiacid $+\mathrm{H}_{2} \mathrm{O}_{2}$

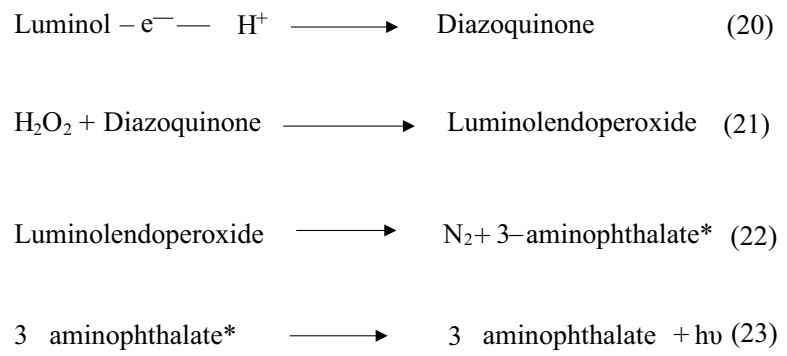

\section{IoT enabled devices}

In everyday life IoT based equipment, viz. digital cameras, mobile phones, webcams, scanners, etc., play an essential role in creating life easy. These physical objects are connected with software, sensors, and other technology for the sole purpose of exchanging data with other systems and devices through the internet. Such devices can readily be applied for easy and accurate detection of analytes for point of care detection in $\mu \mathrm{PADs}$, indicator papers, etc. It is highly advantageous in fact of simplicity, cost, and portability. It provides many opportunities for point of care detection of human health, food/beverages, agriculture, and the environment (Grudpan et al. 2015; Yang et al. 2016; Sharma et al. 2021). Figure 10 illustrates a systematic operational layout of IoT in POCT devices.

\subsection{Scanners}

2-D optical scanners have been used as a low-cost detection system for $\mu$ PADs. It works on diffuse reflectance
Fig. 9 A systematic diagram of a hand-held system for Electrochemiluminescence sensing Reproduced with kind permission from Chen et al. (2016)

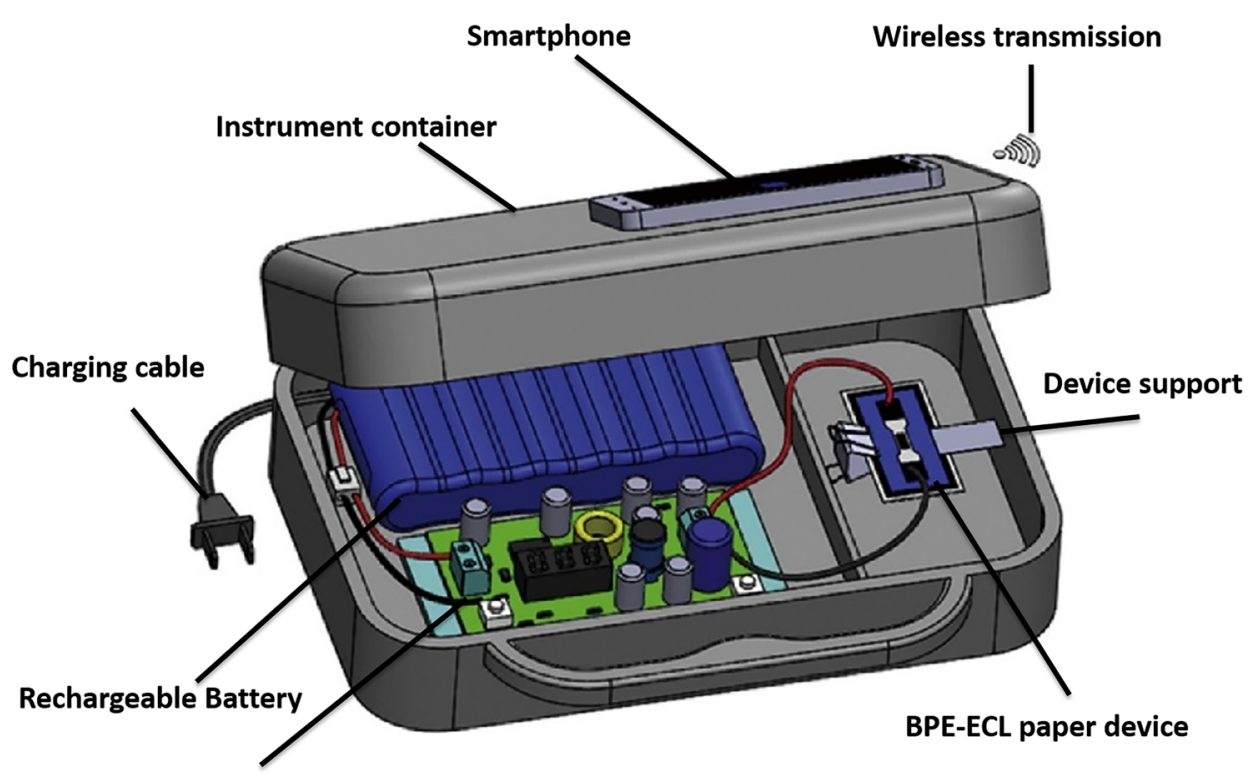

Electronic circuit model 
Fig. 10 Systematic representation of the internet of things in point of care testing

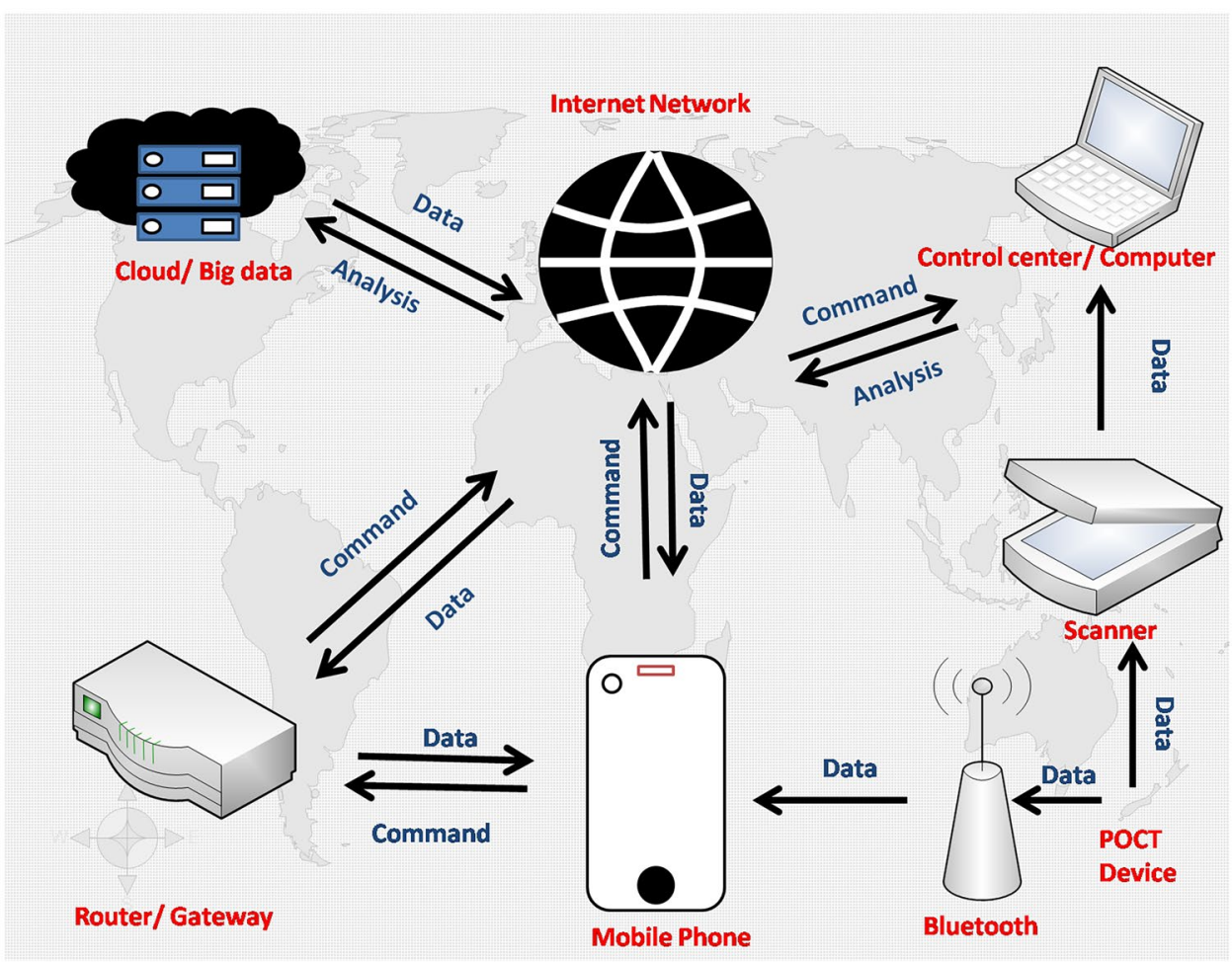

spectroscopy principle, and uses filters for binning light into 3 or 4 wavelengths prior to detection by CCDs instead of separating light. The data generated through the phone camera and scanners is habitually reproved through the device software, which generally hampers the resurgence of the raw data. This pre-proceeding of the data can lead to the difference between colorimetric read-out and can hamper the final result. It is suggested that the issue can be alleviate by getting the raw data through the device, in place of using external scanning calibration through a known object. Frequently, flatbed scanners are preferred for biomedical applications. Because its quantitative analysis is coupled through the $\mu$ PADs, which is favorable and economical for the readouts. In flatbed scanners, illumination condition and the focal distance is constant during the entire single signal readout and also for the consecutive readouts. It generates better images and can be helpful in reproducibility (Grudpan et al. 2015; Göröcs and Ozcan 2014).

\subsection{Smartphones}

Similar to the scanners, smartphones are also used for economical quantitative analysis in paper-based devices. These are portable, light-weighted, and economical, and no special training is required for the operation. The data can be transmitted from direct hardware to the off-site of the specialist, as well as can be directly analyzed through the customized software. It can be utilized for the different analysis methods viz. Colorimetric, fluorescence, electrochemical, etc. It can display the data in the form of a chart, figure, numeric, and in the form of a text message. A wide display, high processor, and huge storage help to qualitative and quantitative analysis of data. It can directly send the command through the internet, which can be further analyzed through cloud storage, and the patient/ specialist can get the qualitative and quantitative data in no time at the remote location.

The current decade is the witness of the development of $\mu$ PADs inbuilt with smart-phones and IoT platform which are frequently used for the point of care testing for human health (Tsagkaris et al. 2021; Kou et al. May 2020; Puneeth and Goel 2021; Ulep et al. 2020; Bagal-Kestwal and Chiang 2021; Kim et al. 2020; Huang et al. 2021; Xu and Yang 2020), food and beverages (Guzman et al. 2018), water quality (Arsawiset and Teepoo 2020; da Silva et al. 2020; Feng et al. 2021), and heavy metal ions (Wang et al. 2020b; Maruthupandi et al. 2020; M. de et al. 2021; Monisha et al. 2021). Recent progress shows various technical advantages and cost-effectiveness of diagnosing diseases in $\mu \mathrm{PADs}$, through a smartphone. Still, it persists multiple physical and technical limitations can be overcome by better implementing filters, using advanced image processing software, and developing unique platforms (Ulep and Yoon 2018).

\subsection{Limitations of $\mu$ PADs for POCT}

$\mu$ PAD for POCT provides economical solutions for the preliminary level diagnosis. It offers a primary screening tool for healthcare to the end-users. Yet, there exists a lot 
of scopes to improve the performance of these devices because of their varying sensitivity and specificity. It may cause altered results in terms of positive and negative. Due to the portability and rough handling of the POCT device, the test strips and reagents may be exposed to environmental conditions (cold, heat, humidity, etc.). Humidity affects the colorimetric test stripes, altitude affects those tests, which is also sensitive to oxygen (viz. glucose and blood gas tests). Disease metabolites, drugs, and diet can affect the POCT test results as compared to laboratory test results (Nichols 2020). The false-negative result of a home pregnancy test kit causes an increased in pregnancy and sexually transmitted infections (Rahman and Berenson 2013). The home pregnancy test kit's false-positive results lead to significant patient anxiety and pointless interventions (Nakhal et al. 2012). It is also observed that the point of care glucose meter's accuracy and clinical performance is hindered at some level of performance (Watkinson et al. 2012), through the unsuitable glucose meter may overestimate and underestimate the glucose level, which may hamper the insulin dose of the patient (Perera et al. 2011). At-home HIV test kit (OraQuick At-Home HIV test) approved through the US food and drug administration (FDA) shows specificity $(99.98 \%)$ and sensitivity $(92 \%)$ which means among the 12 test 1 test result will be false negative (Arnold 2012). Apart from the fabrication, sensitivity, and specificity, handling of the POCT device by the end-users (domestic or medical services) is the major challenge, which affects the final results of the tests (Manocha and Bhargava 2019). Therefore it is required to evaluate errors and risk of errors in POCT more carefully, and it can be achieved by bearing in mind the whole testing procedure by means of well-designed studies aiming to improve outcomes (Plebani 2009).

\subsection{Possible routes to overcome the limitations of $\mu$ PADs for POCT}

Reported devices have certain limitations when they are being used beyond manufacturers' recommendations. Errors can be occurred due to the number of operators involved, test specimen volume, test locations. The quality of a $\mu$ PADs can be estimated through its specificity and sensitivity, which is dependent on the four main functions (1) Detection methods and various substrates, (2) Methods of result determination, (3) Robustness of testing device (4) Variation in batch production.

Errors in producing accurate results on its multiple usages can be reduced by employing risk management during the development of new methods, by documentation of common hazards, limitations, and instruction for use. Risk management examines each and every step of the testing process for finding the weakness of the procedure where errors may occur (Nichols 2020). The detection methods diverge from optical to electrochemical; for each available detection method, a range of substrates is known for getting the best possible specificity, sensitivity, and cost reduction. The capillary flow speed is a dependent part of the substrate use; as the capillary flow speed increases, the value of specificity increases, and the value of sensitivity decreases simultaneously, directly affecting the test signals. For colorimetric determination, the test result is determined through the naked eye or by the end-user camera. The signal readout is an illumination-dependent term in colorimetric and depends on end-user judgment, mainly when the signal value is near the porch. The robust behavior of the testing devices affects the performance of the devices; the reagents used for the analysis, viz. antigens or antibodies and enzymes, should endure insensitive circumstances during testing, storage, and shipping (Then and Garnier 2013). The testing signals also depend on the atmospheric conditions (temperature, humidity, etc.), directly affecting the sample migration speed and stability of enzymes and reagents, shows variation in end signals. While during batch production, the reproducibility of $\mu$ PADs gets hampered. By keeping these points in mind, the specificity and sensitivity of the $\mu$ PADs can be further improved at clinical and domestic levels. The data of colorimetric analysis is so vulnerable for the heterogeneous color distribution due to the uneven shape of the colored area and noise of structures. This limitation is addressed in the colorimetric method for quantifying accurate absorbance value up to the pixel level through a scanner and smartphone. Accurate and precise data is obtained through the computational process (Soda et al. 2020). In another work, a quantitative relationship is determined between the concentration and color data for getting accurate results (Soda and Bakker 2019). Further sensitivity, accuracy, simplicity, and multi-functionality can be added through the blending of biomedical and material science. We believe that by improving the sensitivity and specificity we can improve the performance of the device, and will reduce the false negative/positive results as well as optimization of the $\mu$ PADs by using theoretical simulation can minimize the sample requirement and will reduce the wastage of sample. We also envision that IoT and machine learning will help in obtaining precise results.

\section{Conclusion and future perspectives}

$\mu$ PADs have been broadly employed to advance POCT due to their low cost and ease of manufacturing. Diagnosis of disease in low-resource settings is challenging due to the unavailability of trained personal and resources. Diagnosis in many countries is generally performed through histopathology assessment, which needs dedicated space, equipment, professional staffing. Conventional mode to conduct 
the initial screening of diseases is performed on the medical centers, which are expensive, time-consuming, and require trained personals for machinery operation. Thus, for the on-site clinical diagnosis, IOT enabled POCT can be a viable solution in the context of portable, economical with reasonable accuracy, and increased sensitivity of the devices. There is no requirement for skilled medical staff and heavy equipment in this case, and it can be performed at home in a remote location. Advance devices, including smartphones, can play a vital role and be a part of sustainable and economical solutions in the enhancement of disease diagnostics. It provides high computing power, high-definition display, and user interface. Along with various facilities, smartphones also offer a high-performance camera with adequately huge numerical aperture, storage capability, ability to transfer images and analysis. With the use of IoT, sustainable, cost-effective, and environmentfriendly POCT devices can be developed, which will help for the initial diagnosis of the diseases.

In this review article, we briefly discussed the application and development of diverse detection platforms of a paper-based microfluidic device for point-of-care testing, with their design layout, surface modification, sensing mechanism, and performances, with various detection methods. These platforms enabled the transition from qualitative analysis, semi- quantification to quantitative detection in the POCT field and consequently promoted the study and development of the POCT. These platforms were demonstrated to show desirable performance in the detection of microfluidic chips under specific assay conditions. Despite the diversity of detection methods and detection speed, dimensions, study purpose, and analytes of interest, all the detection platforms provided convincing and rapid test results within the allowable error range. It is observed that by modifying the surface of the detection zone and electrodes, the accuracy level of the results increases in terms of LOD, linear range, and detection time.

As a concluding remark, this review work summarizes the recently published work on the advanced paper-based analytical devices for point-of-care testing for the initial diagnosis. There is a lot of research scope to develop rapid, secure, sustainable, and low-cost detection of the target analytes with greater accuracy.

Future fabrication techniques should focus on the mass production method of the flexible sensors for the commercial development of POCT devices (Gupta and Pal 2018). For the rapid growth of the device, we expect more advancement in inkjet printing, screen printing, and flexographic printing, etc., for the development of hydrophobic and hydrophilic layers by using different chemical substrates that are economical and eco-friendly as well. We also believe that future research will be focused on reducing the fabrication cost with improvement in sensitivity and reproducibility. The sensitivity and reproducibility is the dependent part of the flow control system. So, for the improvement in the flow control system, it is required to control the flow of the fluid by mechanical, geometric, or chemical means. It is required to optimize the $\mu$ PADs by using theoretical simulation to minimize sample requirement and reduce the wastage of sample. It will also help to analyze flow behavior and concentration distribution of the flowing fluid. Furthermore, the detection methods should be low cost with higher sensitivity for achieving accurate and précised results. Furthermore, the future research scope lies in finding out the sustainable solution in the healthcare domain to explore the device's capability by modifying the design to achieve ultra-sensitivity and the wireless read out using IoT-enabled platform from the end user's perspective.

Acknowledgements Authors gratefully acknowledge Start Research Grant (SRG/2020/001895) provided by Science and Engineering Research Board, Department of Science and Technology, India.

Author contributions Conceptualization, VK and AG; resources, AG.; writing - original draft preparation, VK.; writing, review and editing, SP, UTN, KM; supervision and project administration, AG.

\section{Declarations}

Conflict of interest The authors declare no conflict of interest. The funders had no role in the design of the study; in the collection, analyses, or interpretation of data; in the writing of the manuscript, or in the decision to publish the results.

\section{References}

J.A. Adkins, C.S. Henry, Electrochemical detection in paper-based analytical devices using microwire electrodes. Anal. Chim. Acta 891, 247-254 (2015). https://doi.org/10.1016/j.aca.2015.07.019

J.A. Adkins, K. Boehle, C. Friend, B. Chamberlain, B. Bisha, C.S. Henry, Colorimetric and Electrochemical Bacteria Detection Using Printed Paper- and Transparency-Based Analytic Devices. Anal. Chem. 89(6), 3613-3621 (2017). https://doi.org/10.1021/ acs.analchem.6b05009

Advantec MFS. https://www.advantecmfs.com/default.aspx. Accessed 13 Sept 2021

Ahlstrom-Munksjö - Other specialty papers. https://www.ahlstrommunksjo.com/products/specialty-papers/. Accessed 13 Sept 2021

B. Al Mughairy, H.A.J. Al-Lawati, Recent analytical advancements in microfluidics using chemiluminescence detection systems for food analysis. TrAC - Trends Anal. Chem. 124, 115802 (2020). https://doi.org/10.1016/j.trac.2019.115802

Q. An, M. Yu, Y. Zhang, W. Ma, J. Guo, C. Wang, Fe 30 4@ carbon microsphere supported Ag-Au bimetallic nanocrystals with the enhanced catalytic activity and selectivity for the reduction of nitroaromatic compounds. J. Phys. Chem. C 116(42), 2243222440 (2012). https://doi.org/10.1021/jp307629m

R.R. Anjana et al., S, N-doped carbon dots as a fluorescent probe for bilirubin. Microchim. Acta 185(1), 1-11 (2018). https://doi.org/ 10.1007/s00604-017-2574-8 
C. Arnold, At-home HIV test poses dilemmas and opportunities. Lancet. 380(9847), 1045-1046 (2012). https://doi.org/10.1016/ S0140-6736(12)61585-2

S. Arsawiset, S. Teepoo, Ready-to-use, functionalized paper test strip used with a smartphone for the simultaneous on-site detection of free chlorine, hydrogen sulfide and formaldehyde in wastewater. Anal. Chim. Acta. 1118, 63-72 (2020). https://doi.org/10.1016/j. aca.2020.04.041

A. Arshad, H. Wang, X. Bai, R. Jiang, S. Xu, L. Wang, Colorimetric paper sensor for sensitive detection of explosive nitroaromatics based on Au@ Ag nanoparticles. Spectrochim. Acta - Part A Mol. Biomol. Spectrosc. 206, 16-22 (2019). https://doi.org/10.1016/j. saa.2018.07.095

A. Atwe, A. Gupta, R. Kant, M. Das, I. Sharma, S. Bhattacharya, A novel microfluidic switch for $\mathrm{pH}$ control using Chitosan based hydrogels. Microsyst. Technol. 20(7), 1373-1381 (2014). https:// doi.org/10.1007/S00542-014-2112-0

F. Auzel, Upconversion and anti-stokes processes with $\mathrm{f}$ and $\mathrm{d}$ ions in solids. Chem. Rev. 104(1), 139-173 (2004). https://doi.org/10. $1021 / \mathrm{cr} 020357 \mathrm{~g}$

D.R. Bagal-Kestwal, B.H. Chiang, Portable paper-micro well device composed of agglomerated nano-hematite clusters in enzymehydrogel composite for beta glucan detection using smartphone. Sensors Actuators B Chem. 129836 (2021). https://doi.org/10. 1016/j.snb.2021.129836

A. Bagheri pebdeni, M. Hosseini, Fast and selective whole cell detection of Staphylococcus aureus bacteria in food samples by paper based colorimetric nanobiosensor using peroxidase-like catalytic activity of DNA-Au/Pt bimetallic nanoclusters, Microchem. J. 159, 105475 (2020). https://doi.org/10.1016/j.microc.2020. 105475

A. Baranwal et al., Chitosan: An undisputed bio-fabrication material for tissue engineering and bio-sensing applications. Int. J. Biol. Macromol. 110, Elsevier B.V., 110-123 (2018). https://doi.org/ 10.1016/j.ijbiomac.2018.01.006

D. Calabria, C. Caliceti, M. Zangheri, M. Mirasoli, P. Simoni, A. Roda, Smartphone-based enzymatic biosensor for oral fluid L-lactate detection in one minute using confined multilayer paper reflectometry. Biosens. Bioelectron. 94(February), 124-130 (2017). https://doi.org/10.1016/j.bios.2017.02.053

C.K. Camplisson, K.M. Schilling, W.L. Pedrotti, H.A. Stone, A.W. Martinez, Two-ply channels for faster wicking in paper-based microfluidic devices. Lab Chip 15(23), 4461-4466 (2015). https://doi.org/10.1039/c5lc01115a

L. Cao et al., A disposable paper-based microfluidic immunosensor based on reduced graphene oxide-tetraethylene pentamine/Au nanocomposite decorated carbon screen-printed electrodes. Sensors Actuators, B Chem. 252, 44-54 (2017). https://doi.org/10. 1016/j.snb.2017.05.148

L. Cao, C. Fang, R. Zeng, X. Zhao, Y. Jiang, Z. Chen, Paper-based microfluidic devices for electrochemical immunofiltration analysis of human chorionic gonadotropin. Biosens. Bioelectron. 92(February), 87-94 (2017). https://doi.org/10.1016/j.bios.2017.02.002

L. Cao, G.C. Han, H. Xiao, Z. Chen, C. Fang, A novel 3D paper-based microfluidic electrochemical glucose biosensor based on rGOTEPA/PB sensitive film. Anal. Chim. Acta 1096, 34-43 (2020). https://doi.org/10.1016/j.aca.2019.10.049

D.M. Cate, J.A. Adkins, J. Mettakoonpitak, C.S. Henry, Recent developments in paper-based microfluidic devices. Analytical Chem. 87(1), American Chemical Soc 19-41 (2015). https://doi.org/ $10.1021 / \mathrm{ac} 503968 \mathrm{p}$

S. Chaiyo et al., Non-enzymatic electrochemical detection of glucose with a disposable paper-based sensor using a cobalt phthalocyanine-ionic liquid-graphene composite. Biosens. Bioelectron. 102(August 2017), 113-120 2018. https://doi.org/10.1016/j. bios.2017.11.015
X. Chen et al., A novel naphthofluorescein-based probe for ultrasensitive point-of-care testing of zinc(II) ions and its bioimaging in living cells and zebrafishes. Spectrochim. Acta - Part A Mol. Biomol. Spectrosc. 229, 117949 (2020). https://doi.org/10. 1016/j.saa.2019.117949

$\mathrm{X}$. Chen et al., A paper-supported aptasensor based on upconversion luminescence resonance energy transfer for the accessible determination of exosomes. Biosens. Bioelectron. 102(June 2017), 582-588 (2018). https://doi.org/10.1016/j.bios.2017.12.012

$\mathrm{X}$. Chen et al., Determination of glucose and uric acid with bienzyme colorimetry on microfluidic paper-based analysis devices. Biosens. Bioelectron. 35(1), 363-368 (2012). https://doi.org/10. 1016/j.bios.2012.03.018

L. Chen, C. Zhang, D. Xing, Paper-based bipolar electrode-electrochemiluminescence (BPE-ECL) device with battery energy supply and smartphone read-out: A handheld ECL system for biochemical analysis at the point-of-care level. Sensors Actuators, B Chem. 237, 308-317 (2016). https://doi.org/10.1016/j. snb.2016.06.105

Y. Chen, J. Wang, Z. Liu, X. Wang, X. Li, G. Shan, A simple and versatile paper-based electrochemiluminescence biosensing platform for hepatitis B virus surface antigen detection. Biochem. Eng. J. 129, 1-6 (2018). https://doi.org/10.1016/j.bej.2017.10.012

S.R. Chinnadayyala, J. Park, H.T.N. Le, M. Santhosh, A.N. Kadam, S. Cho, Recent advances in microfluidic paper-based electrochemiluminescence analytical devices for point-of-care testing applications. Biosens. Bioelectron. 126, 68-81 (2019). https://doi.org/10.1016/j. bios.2018.10.038

H.J. Chun, Y.M. Park, Y.D. Han, Y.H. Jang, H.C. Yoon, Paper-based glucose biosensing system utilizing a smartphone as a signal reader. Biochip J. 8(3), 218-226 (2014). https://doi.org/10.1007/ s13206-014-8308-7

F.H. Cincotto, E.L. Fava, F.C. Moraes, O. Fatibello-Filho, R.C. Faria, A new disposable microfluidic electrochemical paper-based device for the simultaneous determination of clinical biomarkers. Talanta 195, 62-68 (2019). https://doi.org/10.1016/j.talan ta.2018.11.022

V.A.O.P. da Silva et al., Microfluidic paper-based device integrated with smartphone for point-of-use colorimetric monitoring of water quality index. Meas. J. Int. Meas. Confed. 164, 108085 (2020). https://doi.org/ 10.1016/j.measurement.2020.108085

Z. Dehghani, M. Hosseini, J. Mohammadnejad, M.R. Ganjali, Novel colorimetric sensor based on peroxidase-like activity of chitosanstabilized Au/Pt nanoclusters for trace lead. Anal. Methods 11(5), 684-690 (2019). https://doi.org/10.1039/c8ay01975d

J.L. Delaney, C.F. Hogan, J. Tian, W. Shen, Electrogenerated chemiluminescence detection in paper-based microfluidic sensors. Anal. Chem. 83(4), 1300-1306 (2011). https://doi.org/10.1021/ac102 $392 \mathrm{t}$

L. Deng, C. Chen, C. Zhu, S. Dong, H. Lu, Multiplexed bioactive paper based on $\mathrm{GO} @ \mathrm{SiO} 2 @ \mathrm{CeO} 2$ nanosheets for a low-cost diagnostics platform. Biosens. Bioelectron. 52, 324-329 (2014). https:// doi.org/10.1016/j.bios.2013.09.005

R. Ding, M. Fiedoruk-Pogrebniak, M. Pokrzywnicka, R. Koncki, J. Bobacka, G. Lisak, Solid reference electrode integrated with paper-based microfluidis for potentiometric ion sensing. Sensors Actuators B. Chem. 128680 (2020). https://doi.org/10.1016/j. snb.2020.128680

Electrochemical Methods: Fundamentals and Applications, 2nd Edition I Wiley. https://www.wiley.com/en-us/Electrochemical+Metho ds $\% 3 \mathrm{~A}+$ Fundamentals+and+Applications $\% 2 \mathrm{C}+2 \mathrm{nd}+$ Edition-p9780471043720. Accessed 17 Aug 2020

E. Elizalde, R. Urteaga, C.L.A. Berli, Rational design of capillarydriven flows for paper-based microfluidics. Lab Chip 15(10), 2173-2180 (2015). https://doi.org/10.1039/c4lc01487a 
E. Evans, E.F. Moreira Gabriel, T.E. Benavidez, W.K. Tomazelli Coltro, C.D. Garcia, Modification of microfluidic paper-based devices with silica nanoparticles. Analyst 139(21), 5560-5567 (2014). https://doi.org/10.1039/c4an01147c

Y. Fan et al., A wireless point-of-care testing system for the detection of neuron-specific enolase with microfluidic paper-based analytical devices. Biosens. Bioelectron. 95(April), 60-66 (2017). https:// doi.org/10.1016/j.bios.2017.04.003

Y. Feng et al., A multifunctional fluorescent probe for visualizing $\mathrm{H} 2 \mathrm{~S}$ in wastewater with portable smartphone via fluorescent paper strip and sensing GSH in vivo. J. Hazard. Mater. 406, 124523 (2021). https://doi.org/10.1016/j.jhazmat.2020.124523

D.C.M. Ferreira et al., Optical paper-based sensor for ascorbic acid quantification using silver nanoparticles. Talanta 141, 188-194 (2015). https://doi.org/10.1016/j.talanta.2015.03.067

F. Figueredo, P.T. Garcia, E. Cortón, W.K.T. Coltro, Enhanced Analytical Performance of Paper Microfluidic Devices by Using Fe3O4 Nanoparticles, MWCNT, and Graphene Oxide. ACS Appl. Mater. Interfaces 8(1), 11-15 (2016). https://doi.org/10. 1021/acsami.5b10027

M. de O.K. Franco, W.T. Suarez, V.B. dos Santos, I.S. Resque, M.H. dos Santos, L.F. Capitán-Vallvey, Microanalysis based on paper device functionalized with cuprizone to determine $\mathrm{Cu} 2+$ in sugar cane spirits using a smartphone. Spectrochim. Acta - Part A Mol. Biomol. Spectrosc. 253, 119580 (2021). https://doi.org/ 10.1016/j.saa.2021.119580

E. Fu, S.A. Ramsey, P. Kauffman, B. Lutz, P. Yager, Transport in twodimensional paper networks. Microfluid. Nanofluidics 10(1), 29-35 (2011). https://doi.org/10.1007/s10404-010-0643-y

L.M. Fu, Y.N. Wang, Detection methods and applications of microfluidic paper-based analytical devices. TrAC - Trends Anal. Chem. 107, 196-211 (2018). https://doi.org/10.1016/j.trac.2018.08.018

E.F.M. Gabriel, P.T. Garcia, T.M.G. Cardoso, F.M. Lopes, F.T. Martins, W.K.T. Coltro, Highly sensitive colorimetric detection of glucose and uric acid in biological fluids using chitosan-modified paper microfluidic devices. Analyst 141(15), 4749-4756 (2016). https:// doi.org/10.1039/c6an00430j

S.C.B. Gopinath, T. Lakshmipriya, Y. Chen, W.M. Phang, U. Hashim, Aptamer-based 'point-of-care testing.' Biotechnol. Adv. 34(3), 198-208 (2016). https://doi.org/10.1016/j.biotechadv.2016.02. 003

Z. Göröcs, A. Ozcan, Biomedical imaging and sensing using flatbed scanners. Lab Chip 14(17), 3248-3257 (2014). https://doi.org/ 10.1039/c4lc00530a

K. Grudpan, S.D. Kolev, S. Lapanantnopakhun, I.D. McKelvie, W. Wongwilai, Applications of everyday IT and communications devices in modern analytical chemistry: A review. Talanta. 136. Elsevier B.V., 84-94 (2015). https://doi.org/10.1016/j. talanta.2014.12.042

L. Guan, J. Tian, R. Cao, M. Li, Z. Cai, W. Shen, Barcode-like paper sensor for smartphone diagnostics: An application of blood typing. Anal. Chem. 86(22), 11362-11367 (2014). https://doi. org/10.1021/ac503300y

A. Gupta, B. Geeta, S. Bhattacharya, Novel Dipstick model for Portable Bio-sensing Application. J. Energy Environ. Sustain. 7, 36-41 (2019). https://doi.org/10.47469/JEES.2019.V07. 100075

A. Gupta, M. Nayak, D. Singh, S. Bhattacharya, Antibody immobilization for $\mathrm{ZnO}$ nanowire based biosensor application. Mater. Res. Soc. Symp. Proc. 1675, 33-39 (2014). https://doi.org/10. 1557/opl.2014.848

A. Gupta, P. Pal, Flexible Sensors for Biomedical Application. Energy, Environ. Sustain. 287-314 (2018). https://doi.org/10. 1007/978-981-10-7751-7_13

A. Gupta, V.K. Patel, C. Pandey, Functional characterization of nano-porous silicate-polymer composite for bovine serum albumin immobilization. Sensors Int. 2, 100080 (2021). https:// doi.org/10.1016/J.SINTL.2021.100080

A. Gupta, V. K. Patel, R. Kant, and S. Bhattacharya, Surface Modification Strategies for Fabrication of Nano-Biodevices. Prog. Adhes. Adhes. 161-185 (2017). https://doi.org/10.1002/9781119407485.CH7

J.M.C.C. Guzman, L.L. Tayo, C.C. Liu, Y.N. Wang, L.M. Fu, Rapid microfluidic paper-based platform for low concentration formaldehyde detection. Sensors Actuators, B Chem. 255, 36233629 (2018). https://doi.org/10.1016/j.snb.2017.09.080

Healthcare Systems Home I GE Healthcare I GE Healthcare (India). https://www.gehealthcare.in/. Accessed 13 Sept 2021

S. Hong, W. Kim, Dynamics of water imbibition through paper channels with wax boundaries. Microfluid. Nanofluidics 19(4), 845853 (2015). https://doi.org/10.1007/s10404-015-1611-3

L. Huang et al., Miniaturized Paper-Based Smartphone Biosensor for Differential Diagnosis of Wild-type Pseudorabies Virus Infection versus Vaccination Immunization. Sensors Actuators. B Chem. 327, 128893 (2021). https://doi.org/10.1016/j.snb.2020.128893

K.K. Hussain, J.M. Moon, D.S. Park, Y.B. Shim, Electrochemical Detection of Hemoglobin: A Review. Electroanalysis 29(10), 2190-2199 (2017). https://doi.org/10.1002/elan.201700308

S.H. Im, K.R. Kim, Y.M. Park, J.H. Yoon, J.W. Hong, H.C. Yoon, An animal cell culture monitoring system using a smartphonemountable paper-based analytical device. Sensors Actuators, B Chem. 229, 166-173 (2016). https://doi.org/10.1016/j.snb. 2016.01.121

B.M. Jayawardane, I.D. McKelvie, S.D. Kolev, Development of a gas-diffusion microfluidic paper-based analytical device $(\mu \mathrm{PAD})$ for the determination of ammonia in wastewater samples. Anal. Chem. 87(9), 4621-4626 (2015). https://doi.org/ 10.1021/acs.analchem.5b00125

S. Jena, A. Gupta, R.K. Pippara, P. Pal, Adit, Wireless Sensing Systems: A Review. Energy. Environ. Sustain. 143-192 (2019). https://doi.org/10.1007/978-981-13-3290-6_9

X.C. Jiang, A.B. Yu, Silver nanoplates: A highly sensitive material toward inorganic anions. Langmuir 24(8), 4300-4309 (2008). https://doi. org/10.1021/la7032252

P. Jiang, M. He, L. Shen, A. Shi, Z. Liu, A paper-supported aptasensor for total IgE based on luminescence resonance energy transfer from upconversion nanoparticles to carbon nanoparticles. Sensors Actuators, B Chem. 239, 319-324 (2017). https:// doi.org/10.1016/j.snb.2016.08.005

Y. Jiao et al., 3D vertical-flow paper-based device for simultaneous detection of multiple cancer biomarkers by fluorescent immunoassay. Sensors Actuators. B Chem. 306, 127239 (2020). https://doi.org/10.1016/j.snb.2019.127239

K. Kaarj, P. Akarapipad, J.Y. Yoon, Simpler, Faster, and Sensitive Zika Virus Assay Using Smartphone Detection of Loopmediated Isothermal Amplification on Paper Microfluidic Chips. Sci. Rep. 8(1), 12438 (2018). https://doi.org/10.1038/ s41598-018-30797-9

D. Kim, S.J. Kim, H.T. Ha, S. Kim, Smartphone-based image analysis coupled to paper-based colorimetric devices. Curr. Appl. Phys. 20(9), 1013-1018 (2020). https://doi.org/10.1016/j.cap.2020.06.021

X. Kou et al., Smartphone-assisted robust enzymes@MOFs-based paper biosensor for point-of-care detection. Biosens. Bioelectron. 156, 112095 (2020). https://doi.org/10.1016/j.bios.2020.112095

W.Y. Li et al., Efficient in situ growth of enzyme-inorganic hybrids on paper strips for the visual detection of glucose. Biosens. Bioelectron. 99, 603-611 (2018). https://doi.org/10.1016/j.bios.2017. 08.015

W. Li et al., Fully-drawn origami paper analytical device for electrochemical detection of glucose. Sensors Actuators, B Chem. 231, 230-238 (2016). https://doi.org/10.1016/j.snb.2016.03.031

F. Li et al., High-resolution temporally resolved chemiluminescence based on double-layered 3D microfluidic paper-based device for 
multiplexed analysis. Biosens. Bioelectron. 141(June), 111472 (2019). https://doi.org/10.1016/j.bios.2019.111472

F. Li et al., Multiplexed chemiluminescence determination of three acute myocardial infarction biomarkers based on microfluidic paper-based immunodevice dual amplified by multifunctionalized gold nanoparticles. Talanta 207, 120346 (2020). https://doi. org/10.1016/j.talanta.2019.120346

X. Li, C. Zhao, X. Liu, A paper-based microfluidic biosensor integrating zinc oxide nanowires for electrochemical glucose detection. Microsystems Nanoeng. 1, 1-7 (2015). https://doi.org/10.1038/ micronano.2015.14

W. Li, M. Chen, W. Li, C. You, J. Wei, L. Zhi, Synthesis of air stable silver nanoparticles and their application as conductive ink on paper based flexible electronics. Mater. Res. Innovations 18, S4723-S4727 (2014). https://doi.org/10.1179/1432891714Z. 000000000867

F. Li, X. Wang, J. Liu, Y. Hu, J. He, Double-layered microfluidic paperbased device with multiple colorimetric indicators for multiplexed detection of biomolecules. Sensors Actuators B Chem. 288, 266273 (2019). https://doi.org/10.1016/j.snb.2019.02.116

W. Liu et al., A molecularly imprinted polymer based a lab-on-paper chemiluminescence device for the detection of dichlorvos. Spectrochim. Acta - Part A Mol. Biomol. Spectrosc. 141, 51-57 (2015). https://doi.org/10.1016/j.saa.2015.01.020

M. Liu et al., An IoT-enabled paper sensor platform for real-time analysis of isothermal nucleic acid amplification tests. Biosens. Bioelectron. 169, 112651 (2020). https://doi.org/10.1016/j.bios. 2020.112651

M.M. Liu et al., MoOx quantum dots with peroxidase-like activity on microfluidic paper-based analytical device for rapid colorimetric detection of $\mathrm{H} 2 \mathrm{O} 2$ released from PC12 cells. Sensors Actuators, B Chem. 305(November 2019), 127512 (2020). https://doi.org/ 10.1016/j.snb.2019.127512

W. Liu, J. Kou, H. Xing, B. Li, Paper-based chromatographic chemiluminescence chip for the detection of dichlorvos in vegetables. Biosens. Bioelectron. 52, 76-81 (2014). https://doi.org/10. 1016/j.bios.2013.08.024

C. Liu, D. Wang, C. Zhang, A novel paperfluidic closed bipolar electrode-electrochemiluminescence sensing platform: Potential for multiplex detection at crossing-channel closed bipolar electrodes. Sensors Actuators, B Chem. 270, 341-352 (2018). https://doi. org/10.1016/j.snb.2018.04.180

A. Määttänen et al., A low-cost paper-based inkjet-printed platform for electrochemical analyses. Sensors Actuators, B Chem. 177, 153-162 (2013). https://doi.org/10.1016/j.snb.2012.10.113

A. Manbohi, S.H. Ahmadi, Sensitive and selective detection of dopamine using electrochemical microfluidic paper-based analytical nanosensor. Sens. Bio-Sensing Res. 23(February), 100270 (2019). https://doi.org/10.1016/j.sbsr.2019.100270

A. Manocha, S. Bhargava, Emerging challenges in point-of-care testing. Curr. Med. Res. Pract. 9(6), 227-230 (2019). https://doi.org/ 10.1016/j.cmrp.2019.11.006

M. Maruthupandi, D. Thiruppathi, N. Vasimalai, One minute synthesis of green fluorescent copper nanocluster: The preparation of smartphone aided paper-based kit for on-site monitoring of nanomolar level mercury and sulfide ions in environmental samples. J. Hazard. Mater. 392, 122294 (2020). https://doi.org/10. 1016/j.jhazmat.2020.122294

M. Moazeni, F. Karimzadeh, A. Kermanpur, Peptide modified paper based impedimetric immunoassay with nanocomposite electrodes as a point-of-care testing of Alpha-fetoprotein in human serum. Biosens. Bioelectron. 117(June), 748-757 (2018). https:// doi.org/10.1016/j.bios.2018.07.016

K. Mondal, A. Gupta, Recent Advances in Carbon-Semiconductor Nanocomposites for Water Remediation. Energy, Environ. Sustain. 45-74 (2018). https://doi.org/10.1007/978-981-10-7551-3_4
K. Mondal, B. Balasubramaniam, A. Gupta, A.A. Lahcen, M. Kwiatkowski, Carbon nanostructures for energy and sensing applications. J. Nanotechnol. 2019, (2019). https://doi.org/10. $1155 / 2019 / 1454327$

Monisha et al., Inkjet-printed paper-based colorimetric sensor coupled with smartphone for determination of mercury $(\mathrm{Hg} 2+)$. J. Hazard. Mater. 414, 125440 (2021). https://doi.org/10.1016/j.jhazm at.2021.125440

R. Nakhal, D. Wood, C. Woodhouse, S. Creighton, False-positive pregnancy tests following enterocystoplasty. BJOG An Int. J. Obstet. Gynaecol. 119(3), 366-368 (2012). https://doi.org/10.1111/j. 1471-0528.2011.03031.x

M. Nayak et al., Integrated sorting, concentration and real time PCR based detection system for sensitive detection of microorganisms. Sci. Rep. 2013 31. 3(1), 1-7 (2013). https://doi.org/10. 1038/srep03266

M.P. Nguyen, N.A. Meredith, S.P. Kelly, C.S. Henry, Design considerations for reducing sample loss in microfluidic paper-based analytical devices. Anal. Chim. Acta 1017, 20-25 (2018). https://doi.org/10.1016/j.aca.2018.01.036

J.H. Nichols, Point-of-care testing, in Contemporary Practice in Clinical Chemistry. (Elsevier, 2020), pp. 323-336

A. Nilghaz, S. Bagherbaigi, C.L. Lam, S.M. Mousavi, E.P. Córcoles, D.H.B. Wicaksono, Multiple semi-quantitative colorimetric assays in compact embeddable microfluidic cloth-based analytical device $(\mu \mathrm{CAD})$ for effective point-of-care diagnostic. Microfluid. Nanofluidics 19(2), 317-333 (2015). https://doi. org/10.1007/s10404-015-1545-9

A. Nilghaz, L. Guan, W. Tan, W. Shen, Advances of Paper-Based Microfluidics for Diagnostics - The Original Motivation and Current Status. ACS Sens. 1(12), 1382-1393 (2016). https://doi.org/ 10.1021/acssensors.6b00578

J. Noiphung, T. Songjaroen, W. Dungchai, C.S. Henry, O. Chailapakul, W. Laiwattanapaisal, Electrochemical detection of glucose from whole blood using paper-based microfluidic devices. Anal. Chim. Acta 788, 39-45 (2013). https://doi.org/10.1016/j.aca.2013.06. 021

G. Palazzo, L. Facchini, A. Mallardi, Colorimetric detection of sugars based on gold nanoparticle formation. Sensors Actuators, B Chem. 161(1), 366-371 (2012). https://doi.org/10.1016/j.snb. 2011.10.046

J. Park, J.K. Park, Pressed region integrated 3D paper-based microfluidic device that enables vertical flow multistep assays for the detection of C-reactive protein based on programmed reagent loading. Sensors Actuators, B Chem. 246, 1049-1055 (2017). https://doi.org/10.1016/j.snb.2017.02.150

C. Parolo, A. de la Escosura-Muñiz, A. Merkoçi, Enhanced lateral flow immunoassay using gold nanoparticles loaded with enzymes. Biosens. Bioelectron. 40(1), 412-416 (2013). https://doi.org/ 10.1016/j.bios.2012.06.049

C. Parolo, A. Merkoçi, Paper-based nanobiosensors for diagnostics. Chem. Soc. Rev. 42(2), 450-457 (2013). https://doi.org/10.1039/ c2cs35255a

S. Parween et al., Cross-linked chitosan biofunctionalized paper-based microfluidic device towards long term stabilization of blood typing antibodies. Int. J. Biol. Macromol. 163, 1233-1239 (2020). https://doi.org/10.1016/j.ijbiomac.2020.07.075

V.K. Patel, A. Gupta, D. Singh, R. Kant, S. Bhattacharya, Surface functionalization to mitigate fouling of biodevices: A critical review. Rev. Adhes. Adhes. 3(4), 444-478 (2015). https://doi. org/10.7569/RAA.2015.097309

N.J. Perera, P.M. Stewart, P.F. Williams, E.L. Chua, D.K. Yue, S. M. Twigg, The danger of using inappropriate point-of-care glucose meters in patients on icodextrin dialysis. Diabet. Med. 28(10), 1272-1276 (2011). https://doi.org/10.1111/j.1464-5491.2011. 03362.x 
M. Plebani, Does POCT reduce the risk of error in laboratory testing? Clin. Chim. Acta. 404(1), 59-64 (2009). https://doi.org/10. 1016/j.cca.2009.03.014

T.N.V.K.V. Prasad, V. Subba Rao Kambala, R. Naidu, A Critical Review on Biogenic Silver Nanoparticles and their Antimicrobial Activity. Curr. Nanosci. 7(4), 531-544 (2011). https://doi. org/10.2174/157341311796196736

M. Puiu, C. Bala, Microfluidics-integrated biosensing platforms as emergency tools for on-site field detection of foodborne pathogens. TrAC - Trends Anal. Chem. 125, 115831 (2020). https:// doi.org/10.1016/j.trac.2020.115831

S.B. Puneeth, S. Goel, Handheld and 'Turnkey' 3D Printed Paper-Microfluidic Viscometer with on-board Microcontroller for Smartphone based Biosensing Applications. Anal. Chim. Acta. 1153, 338303 (2021). https://doi.org/10.1016/j.aca.2021.338303

D. Quesada-González, A. Merkoçi, Mobile phone-based biosensing: An emerging 'diagnostic and communication' technology. Biosens. Bioelectron. 92, Elsevier Ltd, 549-562 (2017). https://doi. org/10.1016/j.bios.2016.10.062

D. Quesada-González, A. Merkoçi, Nanoparticle-based lateral flow biosensors. Biosens. Bioelectron. 73, Elsevier Ltd, 47-63 (2015). https://doi.org/10.1016/j.bios.2015.05.050

M. Rahman, A.B. Berenson, Pregnancy test taking is a correlate of unsafe sex, contraceptive nonadherence, pregnancy, and sexually transmitted infections in adolescent and young adult women. J. Women's Heal. 22(4), 339-343 (2013). https://doi.org/10.1089/ jwh.2012.4029

M. Rahimi-Nasrabadi, F. Mizani, ... M. H.-... A. P. A. M., and undefined 2017, Detection of hydrogen peroxide and glucose by using Tb2 (MoO4) 3 nanoplates as peroxidase mimics, Elsevier, Accessed 03 Jan 2021. [Online]. Available: https://www.sciencedirect.com/ science/article/pii/S1386142517304663

N. Ratnarathorn, O. Chailapakul, C.S. Henry, W. Dungchai, Simple silver nanoparticle colorimetric sensing for copper by paper-based devices. Talanta 99, 552-557 (2012). https://doi.org/10.1016/j. talanta.2012.06.033

N. Ruecha, R. Rangkupan, N. Rodthongkum, O. Chailapakul, Novel paper-based cholesterol biosensor using graphene/polyvinylpyrrolidone/polyaniline nanocomposite. Biosens. Bioelectron. 52, 13-19 (2014). https://doi.org/10.1016/j.bios.2013.08.018

G. Ruiz-Vega et al., Electrochemical POC device for fast malaria quantitative diagnosis in whole blood by using magnetic beads, PolyHRP and microfluidic paper electrodes. Biosens. Bioelectron. 150, 111925 (2020). https://doi.org/10.1016/j.bios.2019.111925

G. Ruiz-Vega, T. Garcia-Berrocoso, J. Montaner, and E. Baldrich, Paper microfluidics on screen-printed electrodes for simple electrochemical magneto-immunosensor performance. Sensors Actuators, B Chem. 298, 126897 (2019). https://doi.org/10. 1016/j.snb.2019.126897

M. Rycenga et al., Controlling the synthesis and assembly of silver nanostructures for plasmonic applications. Chem. Rev. 111(6), 3669-3712 (2011). https://doi.org/10.1021/cr100275d

H. Shafiee et al., Paper and flexible substrates as materials for biosensing platforms to detect multiple biotargets. Sci. Rep. 5(1), 1-9 (2015). https://doi.org/10.1038/srep08719

N. Sharma, A. Nigam, D. Lobanov, A. Gupta, A. Novikov, M. Kumar, Mercury (II) Ion Detection using AgNWs-MoS2 Nanocomposite on GaN HEMT for IoT Enabled Smart Water Quality Analysis. IEEE Internet Things J. (2021). https://doi.org/10.1109/JIOT. 2021.3071382

J. Shen et al., Bimetallic nano-mushrooms with DNA-mediated interior nanogaps for high-efficiency SERS signal amplification. Springer. (2015). https://doi.org/10.1007/s12274-014-0556-2

Y. Soda, E. Bakker, Quantification of Colorimetric Data for Paper-Based Analytical Devices. ACS Senss. 4(12), 3093-3101 (2019). https:// doi.org/10.1021/ACSSENSORS.9B01802
Y. Soda, K.J. Robinson, T.J. Cherubini, E. Bakker, Colorimetric absorbance mapping and quantitation on paper-based analytical devices. Lab Chip. 20(8), 1441-1448 (2020). https://doi.org/10. 1039/D0LC00028K

S.U. Son et al., Naked-eye detection of pandemic influenza a ( $\mathrm{pH} 1 \mathrm{N1}$ ) virus by polydiacetylene (PDA)-based paper sensor as a point-of-care diagnostic platform. Sensors Actuators, B Chem. 291(September 2018), 257-265 (2019). https://doi.org/ 10.1016/j.snb.2019.04.081

A. Soni, S.K. Jha, A paper strip based non-invasive glucose biosensor for salivary analysis. Biosens. Bioelectron. 67, 763-768 (2015). https://doi.org/10.1016/j.bios.2014.09.042

W. T. Sow, F. Ye, C. Zhang, H. Li, Smart materials for point-of-care testing: From sample extraction to analyte sensing and readout signal generator. Biosens. Bioelectron. 170, Elsevier Ltd, 112682 (2020). https://doi.org/10.1016/j.bios.2020.112682

$X$. Sun et al., Ultrasensitive microfluidic paper-based electrochemical/ visual biosensor based on spherical-like cerium dioxide catalyst for miR-21 detection. Biosens. Bioelectron. 105, 218-225 (2018). https://doi.org/10.1016/j.bios.2018.01.025

P.G. Sutariya, H. Soni, S.A. Gandhi, A. Pandya, Single-step fluorescence recognition of $\mathrm{As} 3+, \mathrm{Nd} 3+$ and $\mathrm{Br}-$ using pyrene-linked calix[4]arene: application to real samples, computational modelling and paper-based device. New J. Chem. 43(2), 737-747 (2019). https://doi.org/10.1039/c8nj03506g

Y. Tang, W. Gu, L. Fan, Y. Xu, F. Yin, Y. Liu, Test method study for SVG in wind farm and result analysis. Gaoya Dianqi/high Volt. Appar. 51(6), 15-19 (2015). https://doi.org/10.13296/j.10011609.hva.2015.06.003

C. Te Kung, C.Y. Hou, Y.N. Wang, L.M. Fu, Microfluidic paper-based analytical devices for environmental analysis of soil, air, ecology and river water. Sensors Actuators. B. Chem. 301, 126855 (2019). https://doi.org/10.1016/j.snb.2019.126855

W.L. Then, G. Garnier, Paper diagnostics in biomedicine. Rev. Anal. Chem. 32(4), 269-294 (2013). https://doi.org/10.1515/ revac-2013-0007

T. Tian et al., Integration of target responsive hydrogel with cascaded enzymatic reactions and microfluidic paper-based analytic devices ( $\mu$ PADs) for point-of-care testing (POCT). Biosens. Bioelectron. 77, 537-542 (2016). https://doi.org/10.1016/j.bios. 2015.09.049

A.S. Tsagkaris, D. Migliorelli, L. Uttl, D. Filippini, J. Pulkrabova, J. Hajslova, A microfluidic paper-based analytical device ( $\mu$ PAD) with smartphone readout for chlorpyrifos-oxon screening in human serum. Talanta 222, 121535 (2021). https://doi.org/10. 1016/j.talanta.2020.121535

C.C. Tseng, R.J. Yang, W.J. Ju, L.M. Fu, Microfluidic paper-based platform for whole blood creatinine detection. Chem. Eng. J. 348(April), 117-124 (2018). https://doi.org/10.1016/j.cej.2018.04.191

M. Ueland et al., Capillary-driven microfluidic paper-based analytical devices for lab on a chip screening of explosive residues in soil. J. Chromatogr. A 1436, 28-33 (2016). https://doi.org/10.1016/j. chroma.2016.01.054

T.H. Ulep et al., Smartphone based on-chip fluorescence imaging and capillary flow velocity measurement for detecting ROR $1+$ cancer cells from buffy coat blood samples on dual-layer paper microfluidic chip. Biosens. Bioelectron. 153, 112042 (2020). https:// doi.org/10.1016/j.bios.2020.112042

T.H. Ulep, J.Y. Yoon, Challenges in paper-based fluorogenic optical sensing with smartphones. Nano Converg. 5(1). Korea Nano Technology Research Society. 14 (2018). https://doi.org/10.1186/ s40580-018-0146-1

Y. Wang et al., A novel label-free microfluidic paper-based immunosensor for highly sensitive electrochemical detection of carcinoembryonic antigen. Biosens. Bioelectron. 83, 319-326 (2016). https://doi.org/10.1016/j.bios.2016.04.062 
Y. Wang et al., Label-free microfluidic paper-based electrochemical aptasensor for ultrasensitive and simultaneous multiplexed detection of cancer biomarkers. Biosens. Bioelectron. 136(February), 84-90 (2019). https://doi.org/10.1016/j.bios.2019.04.032

H. Wang et al., Colorimetric fluorescent paper strip with smartphone platform for quantitative detection of cadmium ions in real samples. J. Hazard. Mater. 392, 122506 (2020b). https://doi.org/10. 1016/j.jhazmat.2020.122506

F. Wang et al., Paper-based closed Au-Bipolar electrode electrochemiluminescence sensing platform for the detection of miRNA- 155 . Biosens. Bioelectron. 150, (2020a). https://doi.org/10.1016/j. bios.2019.111917

E.W. Washburn, The dynamics of capillary flow. Phys. Rev. 17(3), 273-283 (1921). https://doi.org/10.1103/PhysRev.17.273

P.J. Watkinson, V.S. Barber, E. Amira, T. James, R. Taylor, J.D. Young, The effects of precision, haematocrit, $\mathrm{pH}$ and oxygen tension on point-of-care glucose measurement in critically ill patients: A prospective study. Ann. Clin. Biochem. 49(2), 144-151 (2012). https://doi.org/10.1258/acb.2011.011162

B. Wei, K. Mao, N. Liu, M. Zhang, Z. Yang, Graphene nanocomposites modified electrochemical aptamer sensor for rapid and highly sensitive detection of prostate specific antigen. Biosens. Bioelectron. 121(August), 41-46 (2018). https://doi.org/10.1016/j.bios. 2018.08.067

L. Wu et al., A paper-based electrochemiluminescence electrode as an aptamer-based cytosensor using PtNi@carbon dots as nanolabels for detection of cancer cells and for in-situ screening of anticancer drugs. Microchim. Acta 183(6), 1873-1880 (2016). https:// doi.org/10.1007/s00604-016-1827-2

Z. Xu, Z. Liu, M. Xiao, L. Jiang, C. Yi, A smartphone-based quantitative point-of-care testing (POCT) system for simultaneous detection of multiple heavy metal ions. Chem. Eng. J. 394, 124966 (2020). https://doi.org/10.1016/j.cej.2020.124966

J. Xu, L. Yang, A dual-readout paper-based sensor for on-site detection of penicillinase with a smartphone, in MicroTAS 2020 - 24th
International Conference on Miniaturized Systems for Chemistry and Life Sciences. 335, 522-523 (2020). https://doi.org/10. 1016/j.snb.2021.129707

N. Yang, C. Chen, P. Wang, J. Sun, H. Mao, Structure optimization method of microfluidic paper chip based on image grey-level statistics for chromogenic reaction. Chem. Eng. Process. - Process Intensif. 143, 107627 (2019). https://doi.org/10.1016/j.cep.2019.107627

K. Yang, H. Peretz-Soroka, Y. Liu, F. Lin, Novel developments in mobile sensing based on the integration of microfluidic devices and smartphones. Lab on a Chip. 16(6). Royal Society of Chemistry, 943-958 (2016). https://doi.org/10.1039/c5lc01524c

A.K. Yetisen et al., Paper-based microfluidic system for tear electrolyte analysis. Lab Chip 17(6), 1137-1148 (2017). https://doi.org/10. 1039/c6lc01450j

A. Zamora-Gálvez, E. Morales-Narváez, J. Romero, A. Merkoçi, Photoluminescent lateral flow based on non-radiative energy transfer for protein detection in human serum. Biosens. Bioelectron. 100, 208-213 (2018). https://doi.org/10.1016/j.bios.2017.09.013

J. Zhou et al., ZnSe quantum dot based ion imprinting technology for fluorescence detecting cadmium and lead ions on a three-dimensional rotary paper-based microfluidic chip. Sensors Actuators. B Chem. 305, (2020). https://doi.org/10.1016/j.snb.2019.127462

W.J. Zhu et al., Bienzyme colorimetric detection of glucose with selfcalibration based on tree-shaped paper strip. Sensors Actuators, B Chem. 190, 414-418 (2014). https://doi.org/10.1016/j.snb. 2013.09.007

X. Zhu, J. Huang, J. Liu, H. Zhang, J. Jiang, R. Yu, A dual enzymeinorganic hybrid nanoflower incorporated microfluidic paperbased analytic device ( $\mu$ PAD) biosensor for sensitive visualized detection of glucose. Nanoscale 9(17), 5658-5663 (2017). https://doi.org/10.1039/c7nr00958e

Publisher's Note Springer Nature remains neutral with regard to jurisdictional claims in published maps and institutional affiliations. 